Article

\title{
Implementation of the 2030 Agenda Sustainable Development Goals in Spain
}

\author{
Alejandra Boto-Álvarez ${ }^{1}\left[\right.$ and Roberto García-Fernández ${ }^{2, *}$ (]) \\ 1 Department of Public Law, Faculty of Law, University of Oviedo, Avenida del Cristo, s/n, 33071 Oviedo, \\ Spain; botoalejandra@uniovi.es \\ 2 Department of Accounting, Faculty of Economics and Business, University of Oviedo, Avenida del Cristo, \\ s/n, 33071 Oviedo, Spain \\ * Correspondence: rgarfer@uniovi.es; Tel.: +34-985-10-39-01
}

Received: 10 February 2020; Accepted: 21 March 2020; Published: 24 March 2020

\begin{abstract}
This paper examines the implementation status of sustainable development goals (SDG) in Spain and explores the extent to which the country will be able to meet European standards in sustainability by the year 2030 within the current regulation and praxis. Based on data retrieved from official statistics supplied by Eurostat for a set of indicators useful to monitor the goals our calculations prognosticate whether Spain will reach the European Union average values. The display of each relevant indicator is provided, as well as discussion on their evolution and some recommendations for an effective implementation of SDG on the mid-term, notwithstanding the peculiar political and socio-economic situation in the country. The study proves that Spain needs to adopt urgent regulatory measures and public policies in order to fulfill its commitment to the 2030 Agenda. Otherwise, if the ongoing trend continues, most of the Spanish indicators will not reach the European average values in the overwhelming majority of the goals, including areas as relevant as the struggle for education or environment.
\end{abstract}

Keywords: sustainable development goals (SDG); agenda 2030; taxation; sustainability; Spain; European Union

\section{Introduction}

Following the examples of other national studies [1,2], this paper sets as its aim to reflect on the implementation status of SDG in Spain in order to assess the actual situation and the future challenges upon real facts and statistical estimations, beyond formal declarations. The final purpose of the study is to display data and provide arguments to question the effectiveness of current regulations, political action and administrative praxis to examine whether ongoing trends are enough to fulfill international commitments to sustainability.

As it is widely known, in the XXI century the traditional approach to development is no longer fit for the new geopolitical and economic framework. In order to "leave no one behind", the three dimensions of sustainable development have to be considered: social development, economic growth and environmental protection [3].

Thus, in September 2015, the Heads of State and Government of 193 countries, meeting at the 70th General Assembly of United Nations (UN), agreed upon the 2030 Agenda for Sustainable Development. It is a plan of action for people, planet and prosperity that seeks to eradicate poverty and hunger everywhere; to combat inequalities within and among countries; to build peaceful, just, and inclusive societies; to protect human rights and promote gender equality and the empowerment of women and girls; and to ensure the lasting protection of the planet and its natural resources [4]. 
The 2030 Agenda itself includes a political Declaration and a set of 17 Sustainable Development Goals (SDG) and 169 targets. Nevertheless, it is indivisible and it must be implemented as a whole, in an integrated rather than a fragmented manner, as the different goals and targets are closely interlinked [5]. In fact, some studies have explored the causal relationships within SDG [6].

Come as it may, the Agenda does not only contain goals and targets, it also tackles the challenge of implementation and, finally yet importantly, a framework for follow up and review. This is crucial and requires customized studies, as general indexes do not always seem to be the best indicators to measure the real progress of the 2030 Agenda [7]. Actually, national-based studies are needed in order to explore public policies coherence with 2030 Agenda [8].

The UN Resolution states that, despite the support and help of international organizations, the successful completion of the SGD is a national responsibility. As a cohesion actor the European Union (EU) has played an active role to implement the SDG in development cooperation with partner countries and provides relevant statistics and indicators that can be used, as this study does, to assess and predict the measure in which a country may reach SGD and its relative position within an international framework, in this case the EU.

Institutionally speaking, Spain is committed to SGD at the highest level. Political engagement to the fulfillment of the 2030 Agenda has been materialized among others in the Cabinet's "Plan for the implementation of the 2030 Agenda", approved in June 2018 [9] and in the creation of the "Sustainable Development Council" in February 2019 [10]. As a matter of fact, Spain was part of the 2018 voluntary national review of the High-level political forum on sustainable development, identifying the 2030 Agenda not only as a compulsory new social contract but also as a great opportunity for the country. Indeed, the report shows how the analysis of the economic crisis, and of its persistent consequences, does teach relevant lessons. There is a need to modify manufacturing patterns; join a profound ecological environmental transformation; ensure that economic growth gains contribute to reduce poverty and improve equality; and protect human rights and social State under the rule of law [11]. On September 25, 2019, Spanish acting President spoke during the SDG Summit celebrated in the UN Headquarters in New York, defining SDG as the roadmap of the Government [12]. In fact, in Spain the policy of sustainable development is carried out relying on strategic documents such as the 2019-2023 National Strategy to Fight Poverty [13]; the Strategic Guide on Energy and Climate, which includes a Draft Bill on Climate Change and Energy Transition, a Just Transition Strategy and an Integrated National Energy and Climate Plan for the period 2021-2030 [14] or the new Urban Agenda on 2030 perspective [15].

Despite that strong institutional wager, the degree in which society is really committed to sustainability is still uncertain and so there are the perspectives on real implementation of the SDG in Spain. Actually, in fall 2019, an official campaign named \#ODSéate was launched in order to get the SDG fully known by population. Since then, any institutional communication of the government, social networks included, is portrayed with SDG logo, icons and the emblematic color wheel [16]. The campaign is one of the measures envisaged by the Cabinet's Plan mentioned above, and its very need proves that citizenship does not even know properly the existence of 2030 Agenda yet.

Since the approval of the aforementioned Action Plan for the implementation of the 2030 Agenda by Spanish 2018 Cabinet [9], progress has been limited mainly due to the fragmentation and political instability that has resulted in two electoral processes, first in April and then in November 2019. The new coalition government formed in January 2020, which does not have a parliamentary majority, appears to be deeply concerned about the situation. Therefore, a Declaration facing Climate and Environmental Emergency in Spain has been adopted, as well as a committing to implement 30 lines of action to address it.

Among other research purposes, this paper aims also to contribute to raise awareness of the deep changes involved in SDG, to ensure accountability and to reflect upon the effectiveness of current environmental policies and regulation from a multidisciplinary (legal and economic) approach [17]. 
It does so by assessing the SDG implementation through dynamic analysis methods and by proposing potential ferenda measures for the next future.

\section{Sustainable Development Goals and the Role of the European Union}

As noted in the previous section, 2030 Agenda sets out 17 SDG and 169 targets, integrated and indivisible, which cover economic, social and environmental dimensions [18]:

- Goal 1. End poverty in all its forms everywhere.

- Goal 2. End hunger, achieve food security and improved nutrition, and promote sustainable agriculture.

- Goal 3. Ensure healthy lives and promote well-being for all at all ages.

- Goal 4. Ensure inclusive and equitable quality education and promote lifelong learning opportunities for all.

- Goal 5. Achieve gender equality and empower all women and girls.

- Goal 6. Ensure availability and sustainable management of water and sanitation for all.

- Goal 7. Ensure access to affordable, reliable, sustainable and modern energy for all.

- Goal 8. Promote sustained, inclusive and sustainable economic growth, full and productive employment and decent work for all.

- Goal 9. Build resilient infrastructure, promote inclusive and sustainable industrialization and foster innovation.

- Goal 10. Reduce inequality within and among countries.

- Goal 11. Make cities and human settlements inclusive, safe, resilient, and sustainable.

- Goal 12. Ensure sustainable consumption and production patterns.

- Goal 13. Take urgent action to combat climate change and its impacts.

- Goal 14. Conserve and sustainably use the oceans, seas and marine resources for sustainable development.

- Goal 15. Protect, restore, and promote sustainable use of terrestrial ecosystems, sustainably manage forests, combat desertification, and halt and reverse land degradation, and halt biodiversity loss.

- Goal 16. Promote peaceful and inclusive societies for sustainable development, provide access to justice for all and build effective, accountable, and inclusive institutions at all levels.

- Goal 17. Strengthen the means of implementation and revitalize the Global Partnership for Sustainable Development.

One vital feature of the Agenda is the conception of social and economic development depending on natural resources and environment, and as a matter of shared liability. Thus, persevered cooperation is needed to tackle major scientific and policy issues [19], and such a collective action requires public-private partnerships [20] and multilevel transformations [21]. In current times, agitated by centripetal forces as Brexit, it is an excellent opportunity for EU to strengthen its position as a global power [22], as sustainable development has long been a main goal within the EU integration pathway [23].

Then, not surprisingly, both the European Parliament [24,25] and the EU Council [26,27] have repeatedly invited the European Commission to build an integrated strategy to enforce the 2030 Agenda and the SDG. Following these suggestions, the European Commission today integrates SDG into several European actions and initiatives, and is questioning itself on the best structures, instruments and policies to be approved by EU institutions in order to fulfill the SDG [28].

According to the most recent reviews, it seems that there is still room for improvement [29]. All in all, the importance of regional actors, as the EU, is clear because achieving SDG demands comprehensive policies adjusted to the different dimensions of the territories, economic, social, cultural, and environmental, and operating under a community-based service display [30]. Additionally, stakeholders have also demanded more vertical and horizontal cooperation and dialogue [31]. 
According to official reports [32], all policies, initiatives, and processes ranging from design and shaping to execution, surveillance, reporting, and evaluation should be seen as shared responsibility between different levels of government.

Due to rising globalization, life conditions around the world are more closely interlinked than ever before. Therefore, it is vital to act together on the basis of common beliefs, changing our thinking, procedures and behavior. Economy, social issues and the environment influence one another; there are no more isolated self-contained areas. Economic productivity, social responsibility and concern for natural resources have to be combined. Thus, regional actors with managerial powers in those areas, as the EU, need to assume a leading role, not properly to substitute but to accompany national authorities and to establish a general policy framework.

Regarding assessment and supervision, in 2017, the EU produced an indicator framework as a first step to launch global monitoring, as established in Communication COM (2016) 739 final "Next steps for a sustainable European future". The EU SDG indicator set is aligned in a quite appropriate way with the UN list of global indicators, although there is still an ambition of deeper line-up with the UN SDG indicator list [33]. Some studies [34] have already suggested that the EU should not only harmonize across the EU the efficiency performance and evaluation on the SDG, but also undertake a main task as the prime mover of innovation and knowledge, promoting technical know-how creation.

Academia must also be involved. Indicators are decisive in current governance [35] and thus, research on indicators is crucial for a consistent implementation of the SDG. Indeed the literature review proves that 2030 Agenda is a trending topic, with outstanding papers reflecting on the indicators themselves [7,36,37], providing a sort of achievement ranking based on official public statistics [38] or, more frequently, focusing at specific goals [39-41], fields [42] or geographical regions [1,2,8,43-45]. We are experimenting the effects of the so-called data-revolution [46] and Public Administrations have a prominent role in producing performance information [47].

In Spain, there are objectives and targets for which data have not been published yet by the National Statistical Institute or by other institutions that produce official statistics. Therefore, it is necessary more development in the near future [48]. As some scholars have pointed out, Earth Observation-derived data might be hereby further explored in a near future.

We considered thus useful and relevant for society in general to analyze the forecasts on the degree of Spanish compliance with the SDG, based on the indicators used at EU level and for which Eurostat provides information for the period 2008-2017.

Eurostat is the statistical office of the EU. Its mission is to provide high quality statistics and indicators for Europe. Eurostat is in charge of monitoring the progress to fulfill the 17 SGD and to accomplish this mission it produces a set of 99 indicators (SDGI), 41 of which are multipurpose, that is, they are used to monitor more than one SDG [49]. Assessing Spanish confluence with Europe seems then to be a good quantitative technique to inquire about the achievement of SDG at a national level.

\section{Methodology and Results}

In order to know the measure in which Spain will be able to achieve SDG in 2030, this paper develops a statistical analysis based on the data provided by Eurostat upon some selected indicators for the period 2008-2017. Such a methodology has been already used by other scholars, such as Firoiu et al. to analyze the extent to which Romania could achieve the SDG, taking as reference the average rate of EU [2]. Specifically, we use in first place the forecasting tools provided by Excel program in Microsoft Office 365 (see columns "2020", "2025" and "2030" in Tables 1-17), then the dynamic indexes for each indicator (see columns I2020", "I2025" and "I2030" in Tables 1-17), and finally we analyze whether there might be a confluence point in between the trend designed by the indicators evolution at the level of Spain and that of the matching items at the EU average in year 2030 (see column "CONF" in Tables 1-17). 
Table 1. Sustainable development goal (SDG) 01: End poverty in all its forms everywhere.

\begin{tabular}{|c|c|c|c|c|c|c|c|c|c|c|c|c|c|c|c|c|c|c|}
\hline Indicators & & 2008 & 2009 & 2010 & 2011 & 2012 & 2013 & 2014 & 2015 & 2016 & 2017 & 2020 & 2025 & 2030 & I2020 & I2025 & I2030 & CONF. \\
\hline \multirow{2}{*}{$\begin{array}{l}\text { SDGI } 01.10-\text { People at risk of } \\
\text { poverty or social exclusion (\%) }\end{array}$} & ES & 23.80 & 24.70 & 26.10 & 26.70 & 27.20 & 27.30 & 29.20 & 28.60 & 27.90 & 26.60 & 27.83 & 29.88 & 31.93 & 0.97 & 1.04 & 1.12 & \multirow{2}{*}{$\mathrm{NO}$} \\
\hline & EU & 23.70 & 23.30 & 23.80 & 24.30 & 24.80 & 24.60 & 24.40 & 23.80 & 23.50 & 22.40 & 22.21 & 21.91 & 21.60 & 0.93 & 0.92 & 0.91 & \\
\hline \multirow{2}{*}{$\begin{array}{l}\text { SDGI } 01.20-\text { People at risk of } \\
\text { income poverty after social } \\
\text { transfers }(\%)\end{array}$} & ES & 19.80 & 20.40 & 20.70 & 20.60 & 20.80 & 20.40 & 22.20 & 22.10 & 22.30 & 21.60 & 22.79 & 24.04 & 25.29 & 1.03 & 1.09 & 1.14 & \multirow[b]{2}{*}{$\mathrm{NO}$} \\
\hline & EU & 16.60 & 16.40 & 16.50 & 16.90 & 16.80 & 16.70 & 17.20 & 17.30 & 17.30 & 16.90 & 17.67 & 17.81 & 18.37 & 1.02 & 1.03 & 1.06 & \\
\hline \multirow{2}{*}{$\begin{array}{l}\text { SDGI } 01.30-\text { Severely materially } \\
\text { deprived people }(\%)\end{array}$} & ES & 3.60 & 4.50 & 4.90 & 4.50 & 5.80 & 6.20 & 7.10 & 6.40 & 5.80 & 5.10 & 5.90 & 7.05 & 8.21 & 0.92 & 1.10 & 1.28 & \multirow{2}{*}{$\mathrm{NO}$} \\
\hline & EU & 8.50 & 8.20 & 8.40 & 8.80 & 9.90 & 9.60 & 8.90 & 8.10 & 7.50 & 6.60 & 6.17 & 5.46 & 4.75 & 0.76 & 0.67 & 0.59 & \\
\hline \multirow{2}{*}{$\begin{array}{l}\text { SDGI 01.40-People living in } \\
\text { households with very low work } \\
\text { intensity (\%) }\end{array}$} & ES & 6.60 & 7.60 & 10.80 & 13.40 & 14.30 & 15.70 & 17.10 & 15.40 & 14.90 & 12.80 & 15.39 & 19.70 & 24.02 & 1.00 & 1.28 & 1.56 & \multirow{2}{*}{ NO } \\
\hline & EU & 9.20 & 9.20 & 10.30 & 10.50 & 10.60 & 11.00 & 11.30 & 10.70 & 10.50 & 9.50 & 9.80 & 10.30 & 10.81 & 0.92 & 0.96 & 1.01 & \\
\hline \multirow{2}{*}{$\begin{array}{l}\text { SDGI } 01.41-\text { In work at risk of } \\
\text { poverty rate ( } \% \text { of employed } \\
\text { persons aged } 18 \text { or over) }\end{array}$} & ES & 11.30 & 11.70 & 10.90 & 10.90 & 10.80 & 10.50 & 12.50 & 13.10 & 13.10 & 13.10 & 13.88 & 15.13 & 16.39 & 1.06 & 1.16 & 1.25 & \multirow{2}{*}{$\mathrm{NO}$} \\
\hline & EU & 8.60 & 8.40 & 8.30 & 8.80 & 8.90 & 9.00 & 9.50 & 9.50 & 9.60 & 9.40 & 9.92 & 10.64 & 11.36 & 1.04 & 1.12 & 1.20 & \\
\hline \multirow{2}{*}{$\begin{array}{l}\text { SDGI 01.60-Population living in } \\
\text { a dwelling with a leaking roof, } \\
\text { damp walls, floors or foundation } \\
\text { or rot in window frames of floor } \\
\text { by poverty status (\%) }\end{array}$} & ES & 16.80 & 18.30 & 21.80 & 16.10 & 12.00 & 16.70 & 17.10 & 15.20 & 15.90 & 11.50 & 11.64 & 8.97 & 6.30 & 0.77 & 0.59 & 0.41 & \multirow{2}{*}{ YES } \\
\hline & EU & - & - & - & 15.60 & 15.10 & 15.60 & 15.70 & 15.20 & 15.40 & 13.30 & 13.46 & 12.27 & 11.08 & 0.89 & 0.81 & 0.73 & \\
\hline \multirow{2}{*}{$\begin{array}{l}\text { SDGI } 03.60 \text {-Self-reported unmet } \\
\text { need for medical examination } \\
\text { and care by sex ( } \% \text { of population } \\
\text { aged } 16 \text { and over) }\end{array}$} & ES & 0.40 & 0.50 & 0.30 & 0.60 & 0.70 & 0.80 & 0.60 & 0.60 & 0.50 & 0.10 & 0.12 & 0.09 & 0.05 & 0.20 & 0.14 & 0.08 & \multirow{2}{*}{ YES } \\
\hline & EU & 3.00 & 3.00 & 3.10 & 3.40 & 3.50 & 3.70 & 3.60 & 3.20 & 2.60 & 1.70 & 1.46 & 1.06 & 0.66 & 0.46 & 0.33 & 0.21 & \\
\hline \multirow{2}{*}{$\begin{array}{l}\text { SDGI 06.10-Population having } \\
\text { neither a bath, nor a shower, nor } \\
\text { indoor flushing toilet in their } \\
\text { household by poverty status (\%) }\end{array}$} & ES & 0.10 & - & - & 0.00 & 0.00 & 0.10 & 0.10 & 0.10 & 0.30 & 0.10 & 0.21 & 0.28 & 0.35 & 2.05 & 2.76 & 3.46 & \multirow{2}{*}{ YES } \\
\hline & EU & - & - & - & - & 2.30 & 2.20 & 2.10 & 2.00 & 1.90 & 2.00 & 1.72 & 1.38 & 1.05 & 0.86 & 0.69 & 0.52 & \\
\hline \multirow{2}{*}{$\begin{array}{l}\text { SDGI } 07.60-\text { Population unable } \\
\text { to keep home adequately warm } \\
\text { by poverty status }(\%)\end{array}$} & ES & 5.90 & 7.20 & 7.50 & 6.50 & 9.10 & 8.00 & 11.10 & 10.60 & 10.10 & 8.00 & 11.25 & 13.31 & 15.37 & 1.06 & 1.26 & 1.45 & \multirow{2}{*}{$\mathrm{NO}$} \\
\hline & EU & - & - & 9.50 & 9.80 & 10.80 & 10.70 & 10.30 & 9.40 & 8.70 & 7.80 & 6.62 & 4.57 & 4.40 & 0.70 & 0.49 & 0.27 & \\
\hline \multirow{2}{*}{$\begin{array}{l}\text { SDGI } 11.10-\text { Overcrowding rate } \\
\text { by poverty status }(\%)\end{array}$} & ES & 5.60 & 5.20 & 5.00 & 6.60 & 5.60 & 5.20 & 5.30 & 5.50 & 5.40 & 5.10 & 5.24 & 5.09 & 4.94 & 0.95 & 0.93 & 0.90 & \multirow{2}{*}{ YES } \\
\hline & EU & 18.30 & 17.70 & 17.70 & 17.00 & 16.90 & 17.00 & 16.70 & 16.70 & 16.60 & 15.70 & 15.36 & 14.24 & 13.12 & 0.92 & 0.85 & 0.79 & \\
\hline
\end{tabular}

Source: Eurostat [50] and own estimations. 
Table 2. SDG 02: End hunger, achieve food security and improved nutrition and promote sustainable agriculture.

\begin{tabular}{|c|c|c|c|c|c|c|c|c|c|c|c|c|c|c|c|c|c|c|}
\hline Indicators & & 2008 & 2009 & 2010 & 2011 & 2012 & 2013 & 2014 & 2015 & 2016 & 2017 & 2020 & 2025 & 2030 & I2020 & I2025 & I2030 & CONF. \\
\hline \multirow{2}{*}{$\begin{array}{l}\text { SDGI 02.20-Agricultural factor } \\
\text { income per annual work unit } \\
\text { (Index: } 2010=100 \text { ) (Source: } \\
\text { Eurostat and Directorate-General } \\
\text { for Agriculture and Rural } \\
\text { Development) }\end{array}$} & ES & 94.00 & 94.30 & 100.00 & 101.10 & 102.50 & 112.70 & 118.30 & 125.00 & 135.60 & 133.50 & 150.00 & 175.15 & 200.33 & 1.20 & 1.40 & 1.60 & \multirow{2}{*}{ YES } \\
\hline & EU & 89.50 & 81.10 & 100.00 & 108.80 & 107.50 & 111.90 & 113.60 & 110.00 & 112.50 & 125.20 & 133.85 & 152.31 & 170.80 & 1.22 & 1.38 & 1.55 & \\
\hline \multirow{2}{*}{$\begin{array}{l}\text { SDGI 02.30-Government } \\
\text { support to agricultural research } \\
\text { and development (Million euro) }\end{array}$} & ES & 717.84 & 664.08 & 585.97 & 548.89 & 388.17 & 375.13 & 366.29 & 401.80 & 401.44 & 397.85 & 281.33 & 93.64 & -94.26 & 0.70 & 0.23 & -0.23 & \multirow{2}{*}{ NO } \\
\hline & EU & 3239.53 & 3151.85 & 3215.10 & 3286.98 & 2949.27 & 3048.60 & 2956.84 & 3087.21 & 3141.21 & 3228.48 & 3070.02 & 3016.72 & 2963.36 & 0.99 & 0.98 & 0.96 & \\
\hline \multirow{2}{*}{$\begin{array}{l}\text { SDGI 02.40-Area under organic } \\
\text { farming (\% of utilized } \\
\text { agricultural area) }\end{array}$} & ES & 5.30 & 6.60 & 6.70 & 7.50 & 7.49 & 6.85 & 7.26 & 8.24 & 8.48 & 8.73 & 9.66 & 11.19 & 12.72 & 1.17 & 1.36 & 1.54 & \multirow[b]{2}{*}{ YES } \\
\hline & $\mathrm{EU}$ & 4.30 & 4.70 & 5.10 & 5.40 & 5.66 & 5.70 & 5.78 & 6.20 & 6.68 & 7.03 & 7.91 & 9.38 & 10.85 & 1.28 & 1.51 & 1.75 & \\
\hline \multirow{2}{*}{$\begin{array}{l}\text { SDGI } 02.50-\text { Gross nutrient } \\
\text { balance on agricultural land by } \\
\text { nutrient (kg per hectare) }\end{array}$} & ES & 27.00 & 31.00 & 35.00 & 29.00 & 34.00 & 29.00 & 39.00 & 39.00 & - & - & 42.96 & 49.61 & 56.27 & 1.10 & 1.27 & 1.44 & \multirow[b]{2}{*}{ NO } \\
\hline & $\mathrm{EU}$ & 51.00 & 46.00 & 49.00 & 49.00 & 50.00 & 49.00 & 47.00 & 51.00 & - & - & 49.85 & 50.17 & 50.49 & 0.98 & 0.98 & 0.99 & \\
\hline \multirow{2}{*}{$\begin{array}{l}\text { SDGI 02.60—Ammonia emissions } \\
\text { from agriculture (Ton) (Source: } \\
\text { European Environment Agency) }\end{array}$} & ES & 418,536 & 424,347 & 415,357 & 406,636 & 399,078 & 404,439 & 424,886 & 445,425 & 448,825 & - & $46,224,173$ & 479,003 & $49,578,357$ & 1.04 & 1.08 & 1.11 & \multirow[b]{2}{*}{ NO } \\
\hline & $\mathrm{EU}$ & $3,622,930$ & $3,588,426$ & $3,529,568$ & $3,531,867$ & $3,503,805$ & $3,498,974$ & $3,542,552$ & $3,596,087$ & $3,611,068$ & - & $3,608,975$ & $3,606,361$ & $360,374,367$ & 1.00 & 1.00 & 1.00 & \\
\hline \multirow{2}{*}{$\begin{array}{l}\text { SDGI 06.40-Nitrate in } \\
\text { groundwater (mg NO }{ }_{3} \text { per liter) } \\
\text { (Source: European Environment } \\
\text { Aggency) }\end{array}$} & ES & 40.20 & 37.40 & 37.20 & 38.10 & 39.50 & 39.50 & 39.50 & 39.50 & - & - & 40.34 & 41.15 & 41.97 & 1.02 & 1.04 & 1.06 & \multirow{2}{*}{ NO } \\
\hline & EU & 18.90 & 18.40 & 18.80 & 18.30 & 18.40 & 18.60 & 18.30 & 18.30 & - & - & 18.07 & 18.00 & 17.33 & 0.99 & 0.98 & 0.95 & \\
\hline
\end{tabular}

Source: Eurostat [50] and own estimations. 
Table 3. SDG 03: Ensure healthy lives and promote well-being for all at all ages.

\begin{tabular}{|c|c|c|c|c|c|c|c|c|c|c|c|c|c|c|c|c|c|c|}
\hline Indicators & & 2008 & 2009 & 2010 & 2011 & 2012 & 2013 & 2014 & 2015 & 2016 & 2017 & 2020 & 2025 & 2030 & I 2020 & I2025 & I2030 & CONF. \\
\hline \multirow{2}{*}{$\begin{array}{l}\text { SDGI 03.10-Life expectancy at } \\
\text { birth by sex (years) }\end{array}$} & ES & 81.50 & 81.90 & 82.40 & 82.60 & 82.50 & 83.20 & 83.30 & 83.00 & 83.50 & 83.40 & 84.16 & 85.19 & 86.23 & 1.01 & 1.03 & 1.04 & \multirow[b]{2}{*}{ YES } \\
\hline & EU & 79.40 & 79.60 & 79.90 & 80.20 & 80.30 & 80.50 & 80.90 & 80.60 & 81.00 & 80.90 & 81.56 & 82.44 & 83.32 & 1.01 & 1.02 & 1.03 & \\
\hline \multirow{2}{*}{$\begin{array}{l}\text { SDGI } 03.20-\text { Share of people with } \\
\text { good or very good perceived } \\
\text { health by sex ( } \% \text { of population } \\
\text { aged } 16 \text { or over) }\end{array}$} & ES & 72.50 & 70.80 & 72.00 & 75.50 & 74.40 & 71.70 & 72.90 & 72.60 & 72.50 & 74.20 & 73.92 & 74.51 & 75.10 & 1.02 & 1.03 & 1.03 & \multirow{2}{*}{ YES } \\
\hline & EU & 67.90 & 67.80 & 68.20 & 67.90 & 68.30 & 67.30 & 67.40 & 66.90 & 67.60 & 69.70 & 68.31 & 68.47 & 68.64 & 1.02 & 1.02 & 1.03 & \\
\hline \multirow{2}{*}{$\begin{array}{l}\text { SDGI } 03.40 \text {-Death rate due to } \\
\text { chronic diseases by sex (number } \\
\text { per } 100,000 \text { persons aged less } \\
\text { than } 65 \text { ) }\end{array}$} & ES & 113.00 & 109.60 & 107.00 & 104.40 & 103.20 & 101.00 & 97.40 & 96.40 & - & - & 84.28 & 72.59 & 60.89 & 0.87 & 0.75 & 0.63 & \multirow{2}{*}{ YES } \\
\hline & EU & 143.70 & 139.90 & 135.60 & 132.50 & 130.00 & 126.70 & 123.30 & 122.10 & - & - & 106.23 & 90.56 & 74.88 & 0.87 & 0.74 & 0.61 & \\
\hline \multirow{2}{*}{$\begin{array}{l}\text { SDGI } 03.41-\text { Death rate due to } \\
\text { tuberculosis, HIV and hepatitis by } \\
\text { sex (number per } 100,000 \text { persons) }\end{array}$} & ES & 5.50 & 5.60 & 5.20 & 4.50 & 4.40 & 4.00 & 4.00 & 3.70 & - & - & 2.12 & 0.68 & -0.77 & 0.57 & 0.18 & -0.21 & \multirow{2}{*}{ YES } \\
\hline & EU & 3.90 & 3.70 & 3.60 & 3.40 & 3.30 & 3.10 & 3.00 & 2.90 & - & - & 2.19 & 1.43 & 0.77 & 0.75 & 0.49 & 0.27 & \\
\hline \multirow{2}{*}{$\begin{array}{l}\text { SDGI } 03.60-\text { Self-reported unmet } \\
\text { need for medical examination } \\
\text { and care by sex ( } \% \text { of population } \\
\text { aged } 16 \text { and over) }\end{array}$} & ES & 0.40 & 0.50 & 0.30 & 0.60 & 0.70 & 0.80 & 0.60 & 0.60 & 0.50 & 0.10 & 0.12 & 0.09 & 0.05 & 0.20 & 0.14 & 0.08 & \multirow{2}{*}{ YES } \\
\hline & EU & 3.00 & 3.00 & 3.10 & 3.40 & 3.50 & 3.70 & 3.60 & 3.20 & 2.60 & 1.70 & 1.46 & 1.06 & 0.66 & 0.46 & 0.33 & 0.21 & \\
\hline \multirow{2}{*}{$\begin{array}{l}\text { SDGI } 08.60-\text { People killed in } \\
\text { accidents at work (number per } \\
100,000 \text { employees) }\end{array}$} & ES & 3.33 & 2.58 & 2.19 & 2.50 & 2.16 & 1.88 & 1.93 & 2.30 & 1.92 & - & 1.44 & 0.80 & 0.17 & 0.63 & 0.35 & 0.07 & \multirow[b]{2}{*}{ YES } \\
\hline & EU & 2.39 & 2.01 & 2.11 & 2.05 & 1.95 & 1.80 & 1.82 & 1.83 & 1.71 & - & 1.44 & 1.10 & 0.75 & 0.79 & 0.60 & 0.41 & \\
\hline \multirow{2}{*}{$\begin{array}{l}\text { SDGI 11.20-Population living in } \\
\text { households considering that they } \\
\text { suffer from noise, by poverty } \\
\text { status (\%) }\end{array}$} & ES & 22.00 & 22.40 & 18.40 & 15.60 & 15.00 & 18.30 & 15.90 & 15.70 & 16.20 & 15.20 & 13.12 & 9.67 & 6.21 & 0.84 & 0.62 & 0.40 & \multirow{2}{*}{ YES } \\
\hline & EU & - & - & - & 19.70 & 18.80 & 18.80 & 18.40 & 18.00 & 17.90 & 17.50 & 16.53 & 14.84 & 13.25 & 0.92 & 0.82 & 0.74 & \\
\hline \multirow{2}{*}{$\begin{array}{l}\text { SDGI 11.40-People killed in road } \\
\text { accidents (rate) }\end{array}$} & ES & 6.70 & 5.80 & 5.20 & 4.40 & 4.00 & 3.60 & 3.60 & 3.60 & 3.90 & 3.90 & 3.00 & 1.51 & 0.02 & 0.83 & 0.42 & 0.00 & \multirow[b]{2}{*}{ YES } \\
\hline & EU & 7.90 & 7.00 & 6.20 & 6.10 & 5.60 & 5.10 & 5.10 & 5.10 & 5.00 & - & 3.60 & 1.90 & 0.19 & 0.71 & 0.37 & 0.04 & \\
\hline \multirow{2}{*}{$\begin{array}{l}\text { SDGI } 11.50 \text {-Exposure to air } \\
\text { pollution by particulate matter } \\
\left(\mu \mathrm{g} / \mathrm{m}^{3}\right)\end{array}$} & ES & 14.20 & 15.20 & 12.40 & 12.90 & 13.70 & 10.70 & 11.30 & 13.00 & 11.30 & 12.10 & 10.80 & 8.73 & 9.48 & 0.83 & 0.67 & 0.73 & \multirow[b]{2}{*}{ NO } \\
\hline & EU & 17.50 & 17.40 & 18.10 & 18.40 & 16.80 & 15.70 & 15.20 & 14.60 & 13.80 & 14.10 & 12.50 & 9.96 & 7.42 & 0.86 & 0.68 & 0.51 & \\
\hline
\end{tabular}

Source: Eurostat [50] and own estimations. 
Table 4. SDG 04: Ensure inclusive and equitable quality education and promote lifelong learning opportunities for all.

\begin{tabular}{|c|c|c|c|c|c|c|c|c|c|c|c|c|c|c|c|c|c|c|}
\hline Indicators & & 2008 & 2009 & 2010 & 2011 & 2012 & 2013 & 2014 & 2015 & 2016 & 2017 & 2020 & 2025 & 2030 & I2020 & I2025 & I2030 & CONF. \\
\hline \multirow{2}{*}{$\begin{array}{l}\text { SDGI } 04.10-\text { Early leavers from } \\
\text { education and training by sex (\% } \\
\text { of population aged } 18 \text { to } 24 \text { ) }\end{array}$} & ES & 31.70 & 30.90 & 28.20 & 26.30 & 24.70 & 23.60 & 21.90 & 20.00 & 19.00 & 18.30 & 12.83 & 4.97 & -2.89 & 0.64 & 0.25 & -0.14 & \multirow[b]{2}{*}{ YES } \\
\hline & EU & 14.70 & 14.20 & 13.90 & 13.40 & 12.70 & 11.90 & 11.20 & 11.00 & 10.70 & 10.60 & 10.24 & 9.64 & 9.04 & 0.93 & 0.88 & 0.82 & \\
\hline \multirow{2}{*}{$\begin{array}{l}\text { SDGI } 04.20-\text { Tertiary educational } \\
\text { attainment by sex ( } \% \text { of } \\
\text { population aged } 30 \text { to } 34 \text { ) }\end{array}$} & ES & 41.30 & 40.70 & 42.00 & 41.90 & 41.50 & 42.30 & 42.30 & 40.90 & 40.10 & 41.20 & 40.93 & 40.68 & 40.43 & 1.00 & 0.99 & 0.99 & \multirow[b]{2}{*}{ NO } \\
\hline & EU & 31.10 & 32.30 & 33.80 & 34.80 & 36.00 & 37.10 & 38.00 & 38.70 & 39.20 & 39.90 & 41.90 & 45.23 & 48.56 & 1.08 & 1.17 & 1.25 & \\
\hline \multirow{2}{*}{$\begin{array}{l}\text { SDGI } 04.30-\text { Participation in } \\
\text { early childhood education by sex } \\
\text { (\% of the age group between } \\
4 \text {-years-old and the starting age of } \\
\text { compulsory education) }\end{array}$} & ES & 98.40 & 98.40 & 97.90 & 97.70 & 97.40 & 97.10 & 97.10 & 97.70 & 97.30 & 97.40 & 96.99 & 96.39 & 95.79 & 0.99 & 0.99 & 0.98 & \multirow{2}{*}{ NO } \\
\hline & EU & 91.60 & 92.10 & 92.90 & 93.20 & 93.90 & 94.10 & 94.20 & 94.90 & 95.30 & 95.40 & 96.83 & 98.94 & 101.06 & 1.02 & 1.04 & 1.06 & \\
\hline \multirow{2}{*}{$\begin{array}{l}\text { SDGI } 04.50-\text { Employment rates } \\
\text { of recent graduates by sex ( } \% \text { of } \\
\text { population aged } 20 \text { to } 34 \text { with at } \\
\text { least upper-secondary education) }\end{array}$} & ES & 82.10 & 73.00 & 70.60 & 67.10 & 63.60 & 59.90 & 65.10 & 65.20 & 68.00 & 71.90 & 68.93 & 63.98 & 59.02 & 1.06 & 0.98 & 0.91 & \multirow{2}{*}{ NO } \\
\hline & EU & 82.00 & 78.30 & 77.40 & 77.10 & 75.90 & 75.40 & 76.00 & 76.90 & 78.40 & 80.20 & 79.80 & 79.14 & 78.48 & 1.04 & 1.03 & 1.02 & \\
\hline \multirow{2}{*}{$\begin{array}{l}\text { SDGI } 04.60-\text { Adult participation } \\
\text { in learning by sex ( } \% \text { of } \\
\text { population aged } 25 \text { to } 64 \text { ) }\end{array}$} & ES & 10.70 & 10.80 & 11.20 & 11.20 & 11.20 & 11.40 & 10.10 & 9.90 & 9.40 & 9.90 & 9.36 & 8.55 & 7.75 & 0.95 & 0.86 & 0.78 & \multirow{2}{*}{ NO } \\
\hline & EU & 9.50 & 9.50 & 9.30 & 9.10 & 9.20 & 10.70 & 10.80 & 10.70 & 10.80 & 10.90 & 11.55 & 12.62 & 13.69 & 1.08 & 1.18 & 1.28 & \\
\hline \multirow{2}{*}{$\begin{array}{l}\text { SDGI } 08.20 \text { - Young people } \\
\text { neither in employment nor in } \\
\text { education and training by sex (\% } \\
\text { of population aged } 15 \text { to } 29 \text { ) }\end{array}$} & ES & 13.20 & 15.00 & 15.30 & 15.30 & 15.90 & 16.00 & 15.60 & 15.20 & 14.50 & 13.90 & 13.96 & 14.06 & 14.16 & 0.92 & 0.92 & 0.93 & \multirow[t]{2}{*}{ NO } \\
\hline & EU & 13.10 & 14.80 & 15.20 & 15.40 & 15.90 & 15.90 & 15.30 & 14.80 & 14.20 & 13.40 & 13.33 & 13.22 & 13.10 & 0.90 & 0.89 & 0.89 & \\
\hline
\end{tabular}

Source: Eurostat [50] and own estimations. 
Table 5. SDG 05: Achieve gender equality and empower all women and girls.

\begin{tabular}{|c|c|c|c|c|c|c|c|c|c|c|c|c|c|c|c|c|c|c|}
\hline Indicators & & 2008 & 2009 & 2010 & 2011 & 2012 & 2013 & 2014 & 2015 & 2016 & 2017 & 2020 & 2025 & 2030 & I 2020 & I2025 & I2030 & CONF. \\
\hline \multirow{2}{*}{$\begin{array}{l}\text { SDGI } 05.20-\text { Gender pay gap in } \\
\text { unadjusted form (\% of average } \\
\text { gross hourly earnings of men) }\end{array}$} & ES & 16.10 & 16.70 & 16.20 & 17.60 & 18.70 & 17.80 & 14.90 & 14.20 & 15.10 & 15.10 & 14.36 & 13.18 & 11.99 & 1.01 & 0.93 & 0.84 & \multirow[b]{2}{*}{ YES } \\
\hline & EU & - & - & 17.10 & 17.10 & 17.40 & 16.80 & 16.60 & 16.50 & 16.30 & 16.00 & 15.55 & 14.68 & 13.81 & 0.94 & 0.89 & 0.84 & \\
\hline \multirow{2}{*}{$\begin{array}{l}\text { SDGI } 05.30-\text { Gender employment } \\
\text { gap (percentage points) }\end{array}$} & ES & 19.00 & 14.20 & 12.90 & 11.60 & 10.00 & 9.60 & 10.20 & 11.20 & 11.50 & 11.90 & 10.16 & 7.25 & 4.34 & 0.91 & 0.65 & 0.39 & \multirow[b]{2}{*}{ YES } \\
\hline & EU & 15.10 & 13.50 & 13.00 & 12.80 & 12.20 & 11.70 & 11.50 & 11.60 & 11.60 & 11.50 & 10.43 & 8.70 & 6.97 & 0.90 & 0.75 & 0.60 & \\
\hline \multirow{2}{*}{$\begin{array}{l}\text { SDGI } 05.40 \text {-Inactive population } \\
\text { due to caring responsibilities by } \\
\text { sex (\% of inactive population } \\
\text { aged } 20 \text { to } 64 \text { ) }\end{array}$} & ES & 37.20 & 35.10 & 33.20 & 32.70 & 30.10 & 28.40 & 28.00 & 29.90 & 29.80 & 29.70 & 27.63 & 24.18 & 20.72 & 0.92 & 0.81 & 0.69 & \multirow{2}{*}{ YES } \\
\hline & EU & 20.00 & 19.90 & 19.10 & 19.00 & 19.40 & 20.40 & 20.20 & 20.70 & 21.10 & 21.40 & 22.00 & 23.01 & 24.03 & 1.06 & 1.11 & 1.16 & \\
\hline \multirow{2}{*}{$\begin{array}{l}\text { SDGI } 05.50-\text { Seats held by } \\
\text { women in national parliaments } \\
\text { and governments (Source: } \\
\text { European Institute for Gender } \\
\text { Equality) (\% of seats) }\end{array}$} & ES & 33.30 & 33.80 & 34.60 & 28.70 & 37.20 & 37.20 & 37.70 & 39.90 & 38.30 & 38.80 & 42.38 & 46.42 & 50.46 & 1.06 & 1.16 & 1.26 & \multirow[b]{2}{*}{ YES } \\
\hline & EU & 23.20 & 23.40 & 23.60 & 23.60 & 25.30 & 26.70 & 27.20 & 28.00 & 28.20 & 29.80 & 32.03 & 35.88 & 39.74 & 1.14 & 1.28 & 1.42 & \\
\hline \multirow{2}{*}{$\begin{array}{l}\text { SDGI } 05.60-\text { Positions held by } \\
\text { women in senior management } \\
\text { positions (Source: European } \\
\text { Institute for Gender Equality) (\% } \\
\text { of positions) }\end{array}$} & ES & 8.00 & 9.60 & 9.50 & 11.10 & 12.30 & 14.80 & 16.90 & 18.70 & 20.30 & 22.00 & 26.84 & 34.92 & 43.00 & 1.44 & 1.87 & 2.30 & \multirow[b]{2}{*}{$\mathrm{NO}$} \\
\hline & EU & 10.80 & 11.00 & 11.90 & 13.70 & 15.80 & 17.80 & 20.20 & 22.70 & 23.90 & 25.30 & 30.47 & 39.09 & 47.71 & 1.34 & 1.72 & 2.10 & \\
\hline \multirow{2}{*}{$\begin{array}{l}\text { SDGI } 04.10-\text { Early leavers from } \\
\text { education and training by sex ( } \% \\
\text { of population aged } 18 \text { to } 24 \text { ) }\end{array}$} & ES & 31.70 & 30.90 & 28.20 & 26.30 & 24.70 & 23.60 & 21.90 & 20.00 & 19.00 & 18.30 & 12.83 & 4.97 & -2.89 & 0.64 & 0.25 & -0.14 & \multirow[b]{2}{*}{ YES } \\
\hline & EU & 14.70 & 14.20 & 13.90 & 13.40 & 12.70 & 11.90 & 11.20 & 11.00 & 10.70 & 10.60 & 10.24 & 9.64 & 9.04 & 0.93 & 0.88 & 0.82 & \\
\hline \multirow{2}{*}{$\begin{array}{l}\text { SDGI } 04.20-\text { Tertiary educational } \\
\text { attainment by sex ( } \% \text { of } \\
\text { population aged } 30 \text { to } 34)\end{array}$} & ES & 41.30 & 40.70 & 42.00 & 41.90 & 41.50 & 42.30 & 42.30 & 40.90 & 40.10 & 41.20 & 40.93 & 40.68 & 40.43 & 1.00 & 0.99 & 0.99 & \multirow[b]{2}{*}{$\mathrm{NO}$} \\
\hline & EU & 31.10 & 32.30 & 33.80 & 34.80 & 36.00 & 37.10 & 38.00 & 38.70 & 39.20 & 39.90 & 41.90 & 45.23 & 48.56 & 1.08 & 1.17 & 1.25 & \\
\hline \multirow{2}{*}{$\begin{array}{l}\text { SDGI } 04.50-\text { Employment rates } \\
\text { of recent graduates by sex ( } \% \text { of } \\
\text { population aged } 20 \text { to } 34 \text { with at } \\
\text { least upper-secondary education) }\end{array}$} & ES & 82.10 & 73.00 & 70.60 & 67.10 & 63.60 & 59.90 & 65.10 & 65.20 & 68.00 & 71.90 & 68.93 & 63.98 & 59.02 & 1.06 & 0.98 & 0.91 & \multirow[b]{2}{*}{$\mathrm{NO}$} \\
\hline & EU & 82.00 & 78.30 & 77.40 & 77.10 & 75.90 & 75.40 & 76.00 & 76.90 & 78.40 & 80.20 & 79.80 & 79.14 & 78.48 & 1.04 & 1.03 & 1.02 & \\
\hline
\end{tabular}


Table 6. SDG 06: Ensure availability and sustainable management of water and sanitation for all.

\begin{tabular}{|c|c|c|c|c|c|c|c|c|c|c|c|c|c|c|c|c|c|c|}
\hline Indicators & & 2008 & 2009 & 2010 & 2011 & 2012 & 2013 & 2014 & 2015 & 2016 & 2017 & 2020 & 2025 & 2030 & I2020 & I2025 & I2030 & CONF. \\
\hline \multirow{2}{*}{$\begin{array}{l}\text { SDGI 06.10-Population having } \\
\text { neither a bath, nor a shower, nor } \\
\text { indoor flushing toilet in their } \\
\text { household by poverty status (\%) }\end{array}$} & ES & 0.10 & - & - & 0.00 & 0.00 & 0.10 & 0.10 & 0.10 & 0.30 & 0.10 & 0.21 & 0.28 & 0.35 & 2.05 & 2.76 & 3.46 & \multirow{2}{*}{ YES } \\
\hline & EU & - & - & - & - & 2.30 & 2.20 & 2.10 & 2.00 & 1.90 & 2.00 & 1.72 & 1.38 & 1.05 & 0.86 & 0.69 & 0.52 & \\
\hline \multirow{2}{*}{$\begin{array}{l}\text { SDGI 06.40-Nitrate in } \\
\text { groundwater (Source: European } \\
\text { Environment Agency) (mg NO} \\
\text { per liter) }\end{array}$} & ES & 40.20 & 37.40 & 37.20 & 38.10 & 39.50 & 39.50 & 39.50 & 39.50 & - & - & 40.34 & 41.15 & 41.97 & 1.02 & 1.04 & 1.06 & \multirow{2}{*}{$\mathrm{NO}$} \\
\hline & EU & 18.90 & 18.40 & 18.80 & 18.30 & 18.40 & 18.60 & 18.30 & 18.30 & - & - & 18.07 & 18.00 & 17.33 & 0.99 & 0.98 & 0.95 & \\
\hline \multirow{2}{*}{$\begin{array}{l}\text { SDGI 14.40-Bathing sites with } \\
\text { excellent water quality by locality } \\
\text { (Source: European Environment } \\
\text { Agency) (Coastal } \\
\text { water-number) }\end{array}$} & ES & - & - & - & 1927 & 1926 & 1931 & 1942 & 1948 & 1949 & 1960 & 1975.70 & 2004.58 & 2033.49 & 1.01 & 1.03 & 1.04 & \multirow{2}{*}{ YES } \\
\hline & EU & - & - & - & 15,444 & 15,406 & 15,436 & 14,828 & 14,791 & 14,821 & 14,935 & $14,370.25$ & $13,774.08$ & $13,177.25$ & 0.97 & 0.93 & 0.89 & \\
\hline
\end{tabular}

Source: Eurostat [50] and own estimations. 
Table 7. SDG 07: Ensure access to affordable, reliable, sustainable and modern energy for all.

\begin{tabular}{|c|c|c|c|c|c|c|c|c|c|c|c|c|c|c|c|c|c|c|}
\hline Indicators & & 2008 & 2009 & 2010 & 2011 & 2012 & 2013 & 2014 & 2015 & 2016 & 2017 & 2020 & 2025 & 2030 & I2020 & I2025 & I2030 & CONF. \\
\hline \multirow{2}{*}{$\begin{array}{l}\text { SDGI 07.10-Primary energy } \\
\text { consumption (Million tons of oil } \\
\text { equivalent) }\end{array}$} & ES & 134.44 & 123.37 & 123.32 & 122.96 & 123.39 & 116.05 & 114.18 & 118.58 & 119.23 & 125.63 & 121.85 & 116.83 & 111.81 & 1.03 & 0.99 & 0.94 & \multirow[b]{2}{*}{ NO } \\
\hline & EU & 1696.86 & 1597.41 & 1660.21 & 1600.63 & 1589.36 & 1573.69 & 1511.29 & 1537.48 & 1546.94 & 1561.59 & 1493.56 & 1418.58 & 1343.52 & 0.97 & 0.92 & 0.87 & \\
\hline \multirow{2}{*}{$\begin{array}{l}\text { SDGI 07.11-Final energy } \\
\text { consumption (Million tons of oil } \\
\text { equivalent) }\end{array}$} & ES & 94.83 & 87.78 & 89.09 & 86.48 & 82.83 & 80.73 & 79.19 & 80.35 & 82.46 & 84.33 & 80.36 & 74.32 & 68.28 & 1.00 & 0.92 & 0.85 & \multirow[b]{2}{*}{ YES } \\
\hline & EU & 1180.27 & 1115.76 & 1163.13 & 1110.65 & 1110.62 & 1110.57 & 1065.57 & 1088.27 & 1110.08 & 1122.77 & 1085.27 & 1052.79 & 1020.27 & 1.00 & 0.97 & 0.94 & \\
\hline \multirow{2}{*}{$\begin{array}{l}\text { SDGI 07.20-Final energy } \\
\text { consumption in households per } \\
\text { capita ( } \mathrm{kg} \text { of oil equivalent) }\end{array}$} & ES & 338.00 & 344.00 & 364.00 & 335.00 & 333.00 & 320.00 & 319.00 & 323.00 & 327.00 & 331.00 & 311.48 & 298.14 & 284.78 & 0.96 & 0.92 & 0.88 & \multirow[b]{2}{*}{ YES } \\
\hline & EU & 605.00 & 600.00 & 639.00 & 568.00 & 593.00 & 599.00 & 525.00 & 547.00 & 563.00 & 563.00 & 517.06 & 480.52 & 443.95 & 0.95 & 0.88 & 0.81 & \\
\hline \multirow{2}{*}{$\begin{array}{l}\text { SDGI 07.30-Energy productivity } \\
\text { (Euro per kilogram of oil } \\
\text { equivalent) }\end{array}$} & ES & 7.40 & 7.80 & 7.80 & 7.70 & 7.50 & 8.00 & 8.20 & 8.20 & 8.40 & 8.30 & 8.65 & 9.14 & 9.64 & 1.05 & 1.11 & 1.18 & \multirow[b]{2}{*}{ NO } \\
\hline & EU & 7.10 & 7.20 & 7.10 & 7.40 & 7.50 & 7.60 & 8.00 & 8.10 & 8.20 & 8.30 & 8.78 & 9.53 & 10.27 & 1.08 & 1.18 & 1.27 & \\
\hline \multirow{2}{*}{$\begin{array}{l}\text { SDGI 07.40-Share of renewable } \\
\text { energy in gross final energy } \\
\text { consumption by sector }(\%)\end{array}$} & ES & 10.74 & 12.96 & 13.81 & 13.22 & 14.29 & 15.32 & 16.13 & 16.22 & 17.36 & 17.51 & 19.79 & 23.24 & 26.69 & 1.22 & 1.43 & 1.65 & \multirow[b]{2}{*}{ YES } \\
\hline & EU & 11.32 & 12.58 & 13.12 & 13.37 & 14.68 & 15.40 & 16.19 & 16.72 & 17.02 & 17.52 & 19.67 & 23.12 & 26.58 & 1.18 & 1.38 & 1.59 & \\
\hline \multirow{2}{*}{$\begin{array}{l}\text { SDGI 07.50-Energy import } \\
\text { dependency by products (\% of } \\
\text { imports in total energy } \\
\text { consumption) }\end{array}$} & ES & 81.66 & 79.67 & 77.15 & 76.17 & 72.97 & 70.18 & 72.75 & 72.94 & 71.49 & 73.94 & 70.68 & 65.81 & 60.94 & 0.97 & 0.90 & 0.84 & \multirow{2}{*}{ NO } \\
\hline & EU & 54.61 & 53.65 & 52.64 & 54.23 & 53.69 & 53.31 & 53.58 & 53.88 & 53.78 & 55.13 & 54.67 & 54.95 & 55.24 & 1.01 & 1.02 & 1.03 & \\
\hline \multirow{2}{*}{$\begin{array}{l}\text { SDGI 07.60-Population unable } \\
\text { to keep home adequately warm } \\
\text { by poverty status (\%) }\end{array}$} & ES & 5.90 & 7.20 & 7.50 & 6.50 & 9.10 & 8.00 & 11.10 & 10.60 & 10.10 & 8.00 & 11.25 & 13.31 & 15.37 & 1.06 & 1.26 & 1.45 & \multirow[b]{2}{*}{ NO } \\
\hline & EU & - & - & 9.50 & 9.80 & 10.80 & 10.70 & 10.30 & 9.40 & 8.70 & 7.80 & 7.01 & 5.71 & 4.40 & 0.75 & 0.61 & 0.47 & \\
\hline \multirow{2}{*}{$\begin{array}{l}\text { SDGI 13.20-Greenhouse gas } \\
\text { emissions intensity of energy } \\
\text { consumption (Source: European } \\
\text { Environment Agency and } \\
\text { Eurostat) (Index: } 2010=100 \text { ) } \\
\end{array}$} & ES & 95.00 & 92.10 & 87.40 & 88.70 & 87.80 & 84.70 & 86.50 & 88.50 & 84.00 & - & 80.71 & 75.64 & 70.56 & 0.91 & 0.85 & 0.80 & \multirow[b]{2}{*}{ YES } \\
\hline & EU & 94.90 & 93.70 & 92.50 & 92.00 & 91.80 & 90.50 & 89.10 & 88.70 & 87.50 & - & 84.20 & 79.79 & 75.37 & 0.95 & 0.90 & 0.85 & \\
\hline
\end{tabular}


Table 8. SDG 08 Promote sustained, inclusive and sustainable economic growth, full and productive employment and decent work for all.

\begin{tabular}{|c|c|c|c|c|c|c|c|c|c|c|c|c|c|c|c|c|c|c|}
\hline Indicators & & 2008 & 2009 & 2010 & 2011 & 2012 & 2013 & 2014 & 2015 & 2016 & 2017 & 2020 & 2025 & 2030 & I2020 & I2025 & I2030 & CONF. \\
\hline \multirow{2}{*}{$\begin{array}{l}\text { SDGI 08.10-Real GDP per capita } \\
\text { (Chain linked volumes (2010), } \\
\text { euro per capita) }\end{array}$} & ES & 24,400 & 23,300 & 23,200 & 22,900 & 22,200 & 21,900 & 22,300 & 23,100 & 23,800 & 24,500 & 24,533 & 24,587 & 24,642 & 1.06 & 1.06 & 1.07 & \multirow[b]{2}{*}{ NO } \\
\hline & EU & 26,300 & 25,000 & 25,500 & 25,900 & 25,700 & 25,700 & 26,100 & 26,700 & 27,100 & 27,700 & 28,273 & 29,297 & 30,322 & 1.06 & 1.10 & 1.14 & \\
\hline \multirow{2}{*}{$\begin{array}{l}\text { SDGI } 08.11-\text { Investment share of } \\
\text { GDP by institutional sectors } \% \text { of } \\
\text { GDP) }\end{array}$} & ES & 29.21 & 24.33 & 23.03 & 21.48 & 19.80 & 18.76 & 19.30 & 19.86 & 19.94 & 20.49 & 18.08 & 14.06 & 10.04 & 0.91 & 0.71 & 0.51 & \multirow[b]{2}{*}{ NO } \\
\hline & EU & 22.79 & 20.85 & 20.36 & 20.48 & 20.06 & 19.61 & 19.77 & 20.15 & 20.41 & 20.65 & 20.13 & 19.34 & 18.55 & 1.00 & 0.96 & 0.92 & \\
\hline \multirow{2}{*}{$\begin{array}{l}\text { SDGI } 08.20 \text { - Young people } \\
\text { neither in employment nor in } \\
\text { education and training by sex (\% } \\
\text { of population aged } 15 \text { to } 29 \text { ) }\end{array}$} & ES & 13.20 & 15.00 & 15.30 & 15.30 & 15.90 & 16.00 & 15.60 & 15.20 & 14.50 & 13.90 & 13.96 & 14.06 & 14.16 & 0.92 & 0.92 & 0.93 & \multirow[b]{2}{*}{ NO } \\
\hline & EU & 13.10 & 14.80 & 15.20 & 15.40 & 15.90 & 15.90 & 15.30 & 14.80 & 14.20 & 13.40 & 13.33 & 13.22 & 13.10 & 0.90 & 0.89 & 0.89 & \\
\hline \multirow{2}{*}{$\begin{array}{l}\text { SDGI } 08.30-\text { Employment rate by } \\
\text { sex }(\% \text { of population aged } 20 \text { to } \\
64 \text { ) }\end{array}$} & ES & 68.50 & 64.00 & 62.80 & 62.00 & 59.60 & 58.60 & 59.90 & 62.00 & 63.90 & 65.50 & 64.79 & 63.61 & 62.43 & 1.05 & 1.03 & 1.01 & \multirow[b]{2}{*}{ NO } \\
\hline & EU & 70.20 & 68.90 & 68.50 & 68.60 & 68.40 & 68.40 & 69.20 & 70.10 & 71.10 & 72.20 & 72.99 & 74.29 & 75.60 & 1.04 & 1.06 & 1.08 & \\
\hline \multirow{2}{*}{$\begin{array}{l}\text { SDGI } 08.40-\text { Long-term } \\
\text { unemployment rate by sex (\% of } \\
\text { active population) }\end{array}$} & ES & 2.00 & 4.30 & 7.30 & 8.90 & 11.00 & 13.00 & 12.90 & 11.40 & 9.50 & 7.70 & 9.92 & 13.62 & 17.33 & 0.87 & 1.19 & 1.52 & \multirow[b]{2}{*}{$\mathrm{NO}$} \\
\hline & EU & 2.60 & 3.00 & 3.80 & 4.10 & 4.60 & 5.10 & 5.00 & 4.50 & 4.00 & 3.40 & 3.78 & 4.41 & 5.05 & 0.84 & 0.98 & 1.12 & \\
\hline \multirow{2}{*}{$\begin{array}{l}\text { SDGI } 08.60-\text { People killed in } \\
\text { accidents at work (number per } \\
100,000 \text { employees) }\end{array}$} & ES & 3.33 & 2.58 & 2.19 & 2.50 & 2.16 & 1.88 & 1.93 & 2.30 & 1.92 & - & 1.44 & 0.80 & 0.17 & 0.63 & 0.35 & 0.07 & \multirow[b]{2}{*}{ YES } \\
\hline & EU & 2.39 & 2.01 & 2.11 & 2.05 & 1.95 & 1.80 & 1.82 & 1.83 & 1.71 & - & 1.44 & 1.10 & 0.75 & 0.79 & 0.60 & 0.41 & \\
\hline \multirow{2}{*}{$\begin{array}{l}\text { SDGI } 01.41-\text { In work at risk of } \\
\text { poverty rate ( } \% \text { of employed } \\
\text { persons aged } 18 \text { or over) }\end{array}$} & ES & 11.30 & 11.70 & 10.90 & 10.90 & 10.80 & 10.50 & 12.50 & 13.10 & 13.10 & 13.10 & 13.88 & 15.13 & 16.39 & 1.06 & 1.16 & 1.25 & \multirow{2}{*}{$\mathrm{NO}$} \\
\hline & $\mathbf{E U}$ & 8.60 & 8.40 & 8.30 & 8.80 & 8.90 & 9.00 & 9.50 & 9.50 & 9.60 & 9.40 & 9.92 & 10.64 & 11.36 & 1.04 & 1.12 & 1.20 & \\
\hline \multirow{2}{*}{$\begin{array}{l}\text { SDGI } 05.40-\text { Inactive population } \\
\text { due to caring responsibilities by } \\
\text { sex ( } \% \text { of inactive population } \\
\text { aged } 20 \text { to } 64 \text { ) }\end{array}$} & ES & 37.20 & 35.10 & 33.20 & 32.70 & 30.10 & 28.40 & 28.00 & 29.90 & 29.80 & 29.70 & 27.63 & 24.18 & 20.72 & 0.92 & 0.81 & 0.69 & \multirow{2}{*}{ YES } \\
\hline & EU & 20.00 & 19.90 & 19.10 & 19.00 & 19.40 & 20.40 & 20.20 & 20.70 & 21.10 & 21.40 & 22.00 & 23.01 & 24.03 & 1.06 & 1.11 & 1.16 & \\
\hline \multirow{2}{*}{$\begin{array}{l}\text { SDGI 12.20-Resource } \\
\text { productivity and domestic } \\
\text { material consumption (Euro per } \\
\text { kilogram, chain linked volumes } \\
{[2010] \text { ) }}\end{array}$} & ES & 1.38 & 1.63 & 1.84 & 2.06 & 2.51 & 2.63 & 2.64 & 2.62 & 2.76 & 2.81 & 3.24 & 3.97 & 4.70 & 1.24 & 1.52 & 1.79 & \multirow{2}{*}{ YES } \\
\hline & EU & 1.61 & 1.75 & 1.85 & 1.79 & 1.93 & 1.98 & 1.98 & 2.03 & 2.07 & 2.08 & 2.22 & 2.51 & 2.73 & 1.10 & 1.24 & 1.35 & \\
\hline
\end{tabular}


Table 9. SDG 09: Build resilient infrastructure, promote inclusive and sustainable industrialization and foster innovation.

\begin{tabular}{|c|c|c|c|c|c|c|c|c|c|c|c|c|c|c|c|c|c|c|}
\hline Indicators & & 2008 & 2009 & 2010 & 2011 & 2012 & 2013 & 2014 & 2015 & 2016 & 2017 & 2020 & 2025 & 2030 & I2020 & I2025 & I2030 & CONF. \\
\hline \multirow{2}{*}{$\begin{array}{l}\text { SDGI } 09.10-\text { Gross domestic } \\
\text { expenditure on R\&D by sector (\% } \\
\text { of GDP) }\end{array}$} & ES & 1.32 & 1.35 & 1.35 & 1.33 & 1.29 & 1.27 & 1.24 & 1.22 & 1.19 & 1.20 & 1.14 & 1.05 & 0.95 & 0.93 & 0.86 & 0.78 & \multirow[b]{2}{*}{ NO } \\
\hline & $\mathrm{EU}$ & 1.83 & 1.93 & 1.92 & 1.97 & 2.00 & 2.02 & 2.03 & 2.04 & 2.04 & 2.06 & 2.13 & 2.24 & 2.35 & 1.04 & 1.10 & 1.15 & \\
\hline \multirow{2}{*}{$\begin{array}{l}\text { SDGI 09.20-Employment in } \\
\text { high- and medium-high } \\
\text { technology manufacturing and } \\
\text { knowledge-intensive services (\% } \\
\text { of total employment) }\end{array}$} & ES & 34.90 & 37.20 & 38.70 & 39.40 & 39.90 & 39.80 & 40.10 & 39.90 & 39.80 & 40.00 & 41.31 & 43.49 & 45.68 & 1.04 & 1.09 & 1.14 & \multirow[b]{2}{*}{ NO } \\
\hline & EU & 42.80 & 43.70 & 44.20 & 44.60 & 44.90 & 45.00 & 45.40 & 45.60 & 45.80 & 45.80 & 46.15 & 46.72 & 47.30 & 1.01 & 1.02 & 1.04 & \\
\hline \multirow{2}{*}{$\begin{array}{l}\text { SDGI } 09.30-R \& D \text { personnel by } \\
\text { sector (\% of active population) }\end{array}$} & ES & 0.94 & 0.96 & 0.96 & 0.92 & 0.90 & 0.88 & 0.88 & 0.88 & 0.91 & 0.96 & 0.94 & 0.92 & 0.90 & 1.07 & 1.04 & 1.02 & \multirow[b]{2}{*}{$\mathrm{NO}$} \\
\hline & $\mathrm{EU}$ & 1.05 & 1.05 & 1.08 & 1.11 & 1.13 & 1.15 & 1.17 & 1.21 & 1.24 & 1.28 & 1.35 & 1.48 & 1.61 & 1.12 & 1.22 & 1.33 & \\
\hline \multirow{2}{*}{$\begin{array}{l}\text { SDGI 09.40-Patent applications } \\
\text { to the European Patent Office } \\
\text { (Source: European Patent Office) } \\
\text { (Number) }\end{array}$} & ES & 1443.53 & 1532.81 & 1511.17 & 1480.28 & 1517.85 & 1512.86 & 1513.42 & 1628.73 & 1641.48 & 1654.56 & 1687.43 & 1789.09 & 1890.85 & 1.04 & 1.10 & 1.16 & \multirow{2}{*}{ YES } \\
\hline & $\mathrm{EU}$ & $57,049.74$ & $56,815.26$ & $56,769.59$ & $57,445.66$ & $56,771.67$ & $56,757.14$ & $56,752.99$ & $57,237.39$ & $55,984.19$ & $54,648.82$ & $54,279.34$ & $53,451.75$ & $52,623.27$ & 0.95 & 0.93 & 0.92 & \\
\hline \multirow{2}{*}{$\begin{array}{l}\text { SDGI } 09.50-\text { Share of busses and } \\
\text { trains in total passenger transport } \\
\text { (\% of total inland passenger-km) }\end{array}$} & ES & 19.80 & 18.70 & 17.70 & 19.10 & 19.30 & 19.30 & 17.30 & 18.60 & 18.40 & - & 17.87 & 17.31 & 16.76 & 0.96 & 0.93 & 0.90 & \multirow[b]{2}{*}{ NO } \\
\hline & $\mathrm{EU}$ & 17.10 & 16.30 & 16.50 & 16.80 & 17.10 & 17.60 & 17.40 & 17.30 & 17.10 & - & 17.50 & 17.96 & 18.43 & 1.01 & 1.04 & 1.07 & \\
\hline \multirow{2}{*}{$\begin{array}{l}\text { SDGI 09.60-Share of rail and } \\
\text { inland waterways in total freight } \\
\text { transport (\% of total inland } \\
\text { freight ton-km) }\end{array}$} & ES & 4.80 & 4.00 & 4.60 & 5.00 & 5.30 & 5.30 & 5.90 & 5.80 & 5.30 & 5.10 & 5.51 & 6.13 & 6.74 & 0.95 & 1.06 & 1.16 & \multirow[t]{2}{*}{$\mathrm{NO}$} \\
\hline & $\mathrm{EU}$ & 24.60 & 23.00 & 24.30 & 25.00 & 25.40 & 25.20 & 25.20 & 24.70 & 23.80 & 23.30 & 23.29 & 23.17 & 23.05 & 0.94 & 0.94 & 0.93 & \\
\hline \multirow{2}{*}{$\begin{array}{l}\text { SDGI 12.30-Average } \mathrm{CO}_{2} \\
\text { emissions per km from new } \\
\text { passenger cars (Source: European } \\
\text { Environment Agency, European } \\
\text { Commission-Directorate-General } \\
\text { for Climate Action) }\left(\mathrm{g} \mathrm{CO}_{2} \text { per }\right. \\
\mathrm{km})\end{array}$} & ES & 148.20 & 142.20 & 137.90 & 133.80 & 128.70 & 122.40 & 118.60 & 115.30 & 114.40 & 115.00 & 116.28 & 118.42 & 120.56 & 1.01 & 1.03 & 1.05 & \multirow{2}{*}{ NO } \\
\hline & EU & - & - & - & - & - & - & 123.40 & 119.50 & 118.10 & 118.50 & 113.35 & 105.66 & 97.96 & 0.95 & 0.88 & 0.82 & \\
\hline
\end{tabular}


Table 10. SDG 10: Reduce inequality within and among countries.

\begin{tabular}{|c|c|c|c|c|c|c|c|c|c|c|c|c|c|c|c|c|c|c|}
\hline Indicators & & 2008 & 2009 & 2010 & 2011 & 2012 & 2013 & 2014 & 2015 & 2016 & 2017 & 2020 & 2025 & 2030 & I2020 & I2025 & I2030 & CONF. \\
\hline \multirow{2}{*}{$\begin{array}{l}\text { SDGI 10.10-Purchasing power } \\
\text { adjusted GDP per capita (Real } \\
\text { expenditure per capita [in } \\
\text { purchasing power standard } \\
\text { EU28]) }\end{array}$} & ES & 26,400 & 24,600 & 24,400 & 24,200 & 24,200 & 24,000 & 24,900 & 26,300 & 26,700 & 27,600 & $28,270.91$ & $29,388.48$ & $30,507.27$ & 1.07 & 1.12 & 1.16 & \multirow[b]{2}{*}{ NO } \\
\hline & $\mathrm{EU}$ & 26,100 & 24,500 & 25,500 & 26,200 & 26,600 & 26,800 & 27,700 & 29,100 & 29,300 & 30,000 & $31,628.30$ & $34,389.17$ & $37,153.06$ & 1.09 & 1.18 & 1.28 & \\
\hline \multirow{2}{*}{$\begin{array}{l}\text { SDGI 10.20-Adjusted gross } \\
\text { disposable income of households } \\
\text { per capita (Purchasing power } \\
\text { standard per inhabitant) }\end{array}$} & ES & 18,527 & 18,319 & 17,759 & 17,797 & 17,563 & 17,581 & 18,254 & 19,311 & 19,205 & 19,336 & 19,748 & 20,431 & 21,115 & 1.02 & 1.06 & 1.09 & \multirow[b]{2}{*}{ NO } \\
\hline & EU & 19,625 & 19,255 & 19,706 & 20,006 & 20,414 & 20,476 & 20,971 & 21,872 & 21,868 & 22,185 & 23,201 & 24,868 & 26,536 & 1.06 & 1.14 & 1.21 & \\
\hline \multirow{2}{*}{$\begin{array}{l}\text { SDGI 10.30-Relative median at } \\
\text { risk of poverty gap (\% distance to } \\
\text { poverty threshold) }\end{array}$} & ES & 25.60 & 25.70 & 26.80 & 27.40 & 30.60 & 30.90 & 31.60 & 33.80 & 31.40 & 32.40 & 36.43 & 40.94 & 45.46 & 1.08 & 1.21 & 1.34 & \multirow[b]{2}{*}{ NO } \\
\hline & EU & 21.90 & 22.20 & 22.90 & 23.00 & 23.40 & 23.80 & 24.60 & 24.80 & 25.00 & 24.10 & 25.96 & 27.61 & 29.25 & 1.05 & 1.11 & 1.18 & \\
\hline \multirow{2}{*}{$\begin{array}{l}\text { SDGI 10.41-Income distribution } \\
\text { (Quintile share ratio) }\end{array}$} & ES & 5.60 & 9 & 6.20 & 6.30 & 6.5 & 6.3 & 6.8 & 6.9 & 6.6 & 6.6 & 6.9 & 7.5 & 8.1 & 1.01 & 1.10 & 1.18 & \multirow[b]{2}{*}{$\mathrm{NO}$} \\
\hline & $\mathrm{EU}$ & 5.00 & 4.90 & 4.90 & 5.00 & 5.00 & 5.00 & 5.20 & 5.20 & 5.20 & 5.10 & 5.23 & 5.38 & 5.53 & 1.01 & 1.03 & 1.06 & \\
\hline \multirow{2}{*}{$\begin{array}{l}\text { SDGI 10.50-Income share of the } \\
\text { bottom } 40 \% \text { of the population (\% } \\
\text { of income) }\end{array}$} & ES & 19.80 & 19.50 & 19.20 & 18.90 & 18.70 & 18.90 & 18.20 & 18.20 & 18.40 & 18.50 & 17.93 & 17.14 & 16.34 & 0.99 & 0.94 & 0.90 & \multirow{2}{*}{ NO } \\
\hline & EU & 21.20 & 21.30 & 21.20 & 21.10 & 21.20 & 21.10 & 20.90 & 20.90 & 20.90 & 21.10 & 20.81 & 20.63 & 20.45 & 1.00 & 0.99 & 0.98 & \\
\hline \multirow{2}{*}{$\begin{array}{l}\text { SDGI 10.60-Asylum applications } \\
\text { by state of procedure (number per } \\
\text { million inhabitants) }\end{array}$} & ES & - & - & 55.00 & 64.00 & 50.00 & 92.00 & 117.00 & 314.00 & 335.00 & 709.00 & 950.07 & 1351.64 & 1753.64 & 3.03 & 4.30 & 5.58 & \multirow{2}{*}{ NO } \\
\hline & EU & 305 & 390 & 411 & 523 & 551 & 727 & 1108 & 2467 & 2361 & 1279 & 2667.44 & 3712.95 & 4759.60 & 1.08 & 1.51 & 1.93 & \\
\hline \multirow{2}{*}{$\begin{array}{l}\text { SDGI 17.20—EU financing to } \\
\text { developing countries by financing } \\
\text { source (Source: Organization for } \\
\text { Economic Co-operation and } \\
\text { Development) (million EUR } \\
\text { [current prices]) }\end{array}$} & ES & 20,456 & 9186 & 7800 & 14,472 & 1539 & 6014 & 10,289 & 19,866 & 4258 & 2301 & 8148.15 & -733.75 & -999.80 & 0.41 & -0.04 & -0.05 & \multirow{2}{*}{ NO } \\
\hline & EU & 119,718 & 102,203 & 127,336 & 153,784 & 147,962 & 129,811 & 171,987 & 178,101 & 143,007 & 155,224 & $189,764.26$ & $196,917.58$ & $225,575.64$ & 1.07 & 1.11 & 1.27 & \\
\hline \multirow{2}{*}{$\begin{array}{l}\text { SDGI 17.30-EU imports from } \\
\text { developing countries by country } \\
\text { income groups (million EUR) }\end{array}$} & ES & 76,616 & 51,928 & 66,735 & 75,388 & 80,650 & 77,020 & 79,482 & 77,589 & 74,762 & 88,378 & $90,201.68$ & $99,955.87$ & $109,720.75$ & 1.16 & 1.29 & 1.41 & \multirow{2}{*}{ NO } \\
\hline & EU & 762,471 & 591,995 & 756,791 & 841,518 & 863,783 & 817,475 & 838,537 & 881,805 & 869,710 & 957,870 & $1,013,951.31$ & $1,142,206.86$ & $1,270,603.04$ & 1.15 & 1.30 & 1.44 & \\
\hline
\end{tabular}


Table 11. SDG 11: Make cities and human settlements inclusive, safe, resilient and sustainable.

\begin{tabular}{|c|c|c|c|c|c|c|c|c|c|c|c|c|c|c|c|c|c|c|}
\hline Indicators & & 2008 & 2009 & 2010 & 2011 & 2012 & 2013 & 2014 & 2015 & 2016 & 2017 & 2020 & 2025 & 2030 & I2020 & I2025 & I2030 & CONF. \\
\hline $\begin{array}{l}\text { SDGI } 11.10-\text { Overcrowding rate } \\
\text { by poverty status (\%) }\end{array}$ & $\begin{array}{l}\text { ES } \\
\text { EU }\end{array}$ & $\begin{array}{c}5.60 \\
18.30\end{array}$ & $\begin{array}{c}5.20 \\
17.70\end{array}$ & $\begin{array}{c}5.00 \\
17.70\end{array}$ & $\begin{array}{c}6.60 \\
17.00\end{array}$ & $\begin{array}{c}5.60 \\
16.90\end{array}$ & $\begin{array}{c}5.20 \\
17.00\end{array}$ & $\begin{array}{c}5.30 \\
16.70\end{array}$ & $\begin{array}{c}5.50 \\
16.70\end{array}$ & $\begin{array}{c}5.40 \\
16.60\end{array}$ & $\begin{array}{c}5.10 \\
15.70\end{array}$ & $\begin{array}{c}5.24 \\
15.36\end{array}$ & $\begin{array}{c}5.09 \\
14.24\end{array}$ & $\begin{array}{c}4.94 \\
13.12\end{array}$ & $\begin{array}{l}0.95 \\
0.92\end{array}$ & $\begin{array}{l}0.93 \\
0.85\end{array}$ & $\begin{array}{l}0.90 \\
0.79\end{array}$ & YES \\
\hline \multirow{2}{*}{$\begin{array}{l}\text { SDGI 11.20-Population living in } \\
\text { households considering that they } \\
\text { suffer from noise, by poverty } \\
\text { status (\%) }\end{array}$} & ES & 22.00 & 22.40 & 18.40 & 15.60 & 15.00 & 18.30 & 15.90 & 15.70 & 16.20 & 15.20 & 13.12 & 9.67 & 6.21 & 0.84 & 0.62 & 0.40 & \multirow{2}{*}{ YES } \\
\hline & EU & - & - & - & 19.70 & 18.80 & 18.80 & 18.40 & 18.00 & 17.90 & 17.50 & 16.53 & 14.84 & 13.25 & 0.92 & 0.82 & 0.74 & \\
\hline \multirow{2}{*}{$\begin{array}{l}\text { SDGI 11.40-People killed in road } \\
\text { accidents (Source: European } \\
\text { Commission-Directorate-General } \\
\text { for Mobility and Transport) (rate) }\end{array}$} & ES & 6.70 & 5.80 & 5.20 & 4.40 & 4.00 & 3.60 & 3.60 & 3.60 & 3.90 & 3.90 & 3.00 & 1.51 & 0.02 & 0.83 & 0.42 & 0.00 & \multirow{2}{*}{ YES } \\
\hline & EU & 7.90 & 7.00 & 6.20 & 6.10 & 5.60 & 5.10 & 5.10 & 5.10 & 5.00 & - & 3.60 & 1.90 & 0.19 & 0.71 & 0.37 & 0.04 & \\
\hline \multirow{2}{*}{$\begin{array}{l}\text { SDGI } 11.50 \text {-Exposure to air } \\
\text { pollution by particulate matter } \\
\text { (Source: European Environment } \\
\text { Agency) }\left(\mu \mathrm{g} / \mathrm{m}^{3}\right)\end{array}$} & ES & 14.20 & 15.20 & 12.40 & 12.90 & 13.70 & 10.70 & 11.30 & 13.00 & 11.30 & 12.10 & 10.80 & 8.73 & 9.48 & 0.83 & 0.67 & 0.73 & \multirow[b]{2}{*}{ NO } \\
\hline & EU & 17.50 & 17.40 & 18.10 & 18.40 & 16.80 & 15.70 & 15.20 & 14.60 & 13.80 & 14.10 & 12.50 & 9.96 & 7.42 & 0.86 & 0.68 & 0.51 & \\
\hline \multirow{2}{*}{$\begin{array}{l}\text { SDGI 11.60-Recycling rate of } \\
\text { municipal waste ( } \% \text { of total waste } \\
\text { generated) }\end{array}$} & ES & 39.70 & 33.20 & 29.20 & 26.70 & 29.80 & 32.50 & 30.80 & 30.00 & 33.90 & 33.50 & 32.89 & 31.89 & 30.90 & 1.10 & 1.06 & 1.03 & \multirow[b]{2}{*}{ NO } \\
\hline & EU & 36.50 & 37.50 & 38.30 & 39.20 & 41.10 & 41.70 & 43.40 & 44.70 & 46.00 & 46.40 & 50.30 & 56.17 & 62.04 & 1.13 & 1.26 & 1.39 & \\
\hline \multirow{2}{*}{$\begin{array}{l}\text { SDGI } 01.60-\text { Population living in } \\
\text { a dwelling with a leaking roof, } \\
\text { damp walls, floors or foundation } \\
\text { or rot in window frames of floor } \\
\text { by poverty status (\%) }\end{array}$} & ES & 16.80 & 18.30 & 21.80 & 16.10 & 12.00 & 16.70 & 17.10 & 15.20 & 15.90 & 11.50 & 11.64 & 8.97 & 6.30 & 0.77 & 0.59 & 0.41 & \multirow{2}{*}{ YES } \\
\hline & EU & - & - & - & 15.60 & 15.10 & 15.60 & 15.70 & 15.20 & 15.40 & 13.30 & 13.46 & 12.27 & 11.08 & 0.89 & 0.81 & 0.73 & \\
\hline \multirow{2}{*}{$\begin{array}{l}\text { SDGI } 09.50-\text { Share of busses and } \\
\text { trains in total passenger transport } \\
\text { (\% of total inland passenger-km) }\end{array}$} & ES & 19.80 & 18.70 & 17.70 & 19.10 & 19.30 & 19.30 & 17.30 & 18.60 & 18.40 & - & 17.87 & 17.31 & 16.76 & 0.96 & 0.93 & 0.90 & \multirow[b]{2}{*}{ NO } \\
\hline & EU & 17.10 & 16.30 & 16.50 & 16.80 & 17.10 & 17.60 & 17.40 & 17.30 & 17.10 & - & 17.50 & 17.96 & 18.43 & 1.01 & 1.04 & 1.07 & \\
\hline \multirow{2}{*}{$\begin{array}{l}\text { SDGI } 16.20 \text { - Population } \\
\text { reporting occurrence of crime, } \\
\text { violence or vandalism in their } \\
\text { area by poverty status }(\%)\end{array}$} & ES & 14.70 & 16.40 & 13.00 & 10.80 & 10.10 & 14.20 & 11.90 & 10.00 & 10.30 & 8.70 & 7.13 & 3.98 & 0.82 & 0.71 & 0.40 & 0.08 & \multirow{2}{*}{ YES } \\
\hline & EU & - & - & 14.40 & 14.10 & 13.60 & 14.50 & 14.00 & 13.60 & 13.00 & 12.00 & 11.70 & 10.35 & 9.00 & 0.86 & 0.76 & 0.66 & \\
\hline
\end{tabular}


Table 12. SDG 12: Ensure sustainable consumption and production patterns.

\begin{tabular}{|c|c|c|c|c|c|c|c|c|c|c|c|c|c|c|c|c|c|c|}
\hline Indicators & & 2008 & 2009 & 2010 & 2011 & 2012 & 2013 & 2014 & 2015 & 2016 & 2017 & 2020 & 2025 & 2030 & I2020 & I 2025 & I2030 & CONF. \\
\hline \multirow{2}{*}{$\begin{array}{l}\text { SDGI 12.20-Resource } \\
\text { productivity and domestic } \\
\text { material consumption (Euro per } \\
\text { kilogram, chain linked volumes } \\
\text { [2010]) }\end{array}$} & ES & 1.38 & 1.63 & 1.84 & 2.06 & 2.51 & 2.63 & 2.64 & 2.62 & 2.76 & 2.81 & 3.24 & 3.97 & 4.70 & 1.24 & 1.52 & 1.79 & \multirow[b]{2}{*}{ YES } \\
\hline & $\mathrm{EU}$ & 1.61 & 1.75 & 1.85 & 1.79 & 1.93 & 1.98 & 1.98 & 2.03 & 2.07 & 2.08 & 2.22 & 2.51 & 2.73 & 1.10 & 1.24 & 1.35 & \\
\hline \multirow{2}{*}{$\begin{array}{l}\text { SDGI 12.30-Average } \mathrm{CO}_{2} \\
\text { emissions per km from new } \\
\text { passenger cars (Source: European } \\
\text { Environment Agency, European } \\
\text { Commission-Directorate-General } \\
\text { for Climate Action) }\left(\mathrm{g} \mathrm{CO}_{2} \text { per }\right. \\
\mathrm{km} \text { ) }\end{array}$} & ES & 148.20 & 142.20 & 137.90 & 133.80 & 128.70 & 122.40 & 118.60 & 115.30 & 114.40 & 115.00 & 116.28 & 118.42 & 120.56 & 1.01 & 1.03 & 1.05 & \multirow{2}{*}{ NO } \\
\hline & EU & - & - & - & - & - & - & 123.40 & 119.50 & 118.10 & 118.50 & 113.35 & 105.66 & 97.96 & 0.95 & 0.88 & 0.82 & \\
\hline \multirow{2}{*}{$\begin{array}{l}\text { SDGI 12.41-Circular material } \\
\text { use rate (\% of material input for } \\
\text { domestic use) }\end{array}$} & ES & - & - & 10.40 & 9.80 & 9.80 & 8.90 & 7.70 & 7.60 & 8.20 & - & 5.72 & 3.38 & 1.03 & 0.75 & 0.44 & 0.14 & \multirow[b]{2}{*}{ NO } \\
\hline & $\mathrm{EU}$ & 9.60 & 10.80 & 11.00 & 10.60 & 11.30 & 11.50 & 11.40 & 11.40 & 11.70 & - & 12.49 & 13.48 & 14.48 & 1.10 & 1.18 & 1.27 & \\
\hline \multirow{2}{*}{$\begin{array}{l}\text { SDGI 07.10-Primary energy } \\
\text { consumption (Million tons of oil } \\
\text { equivalent) }\end{array}$} & ES & 134.44 & 123.37 & 123.32 & 122.96 & 123.39 & 116.05 & 114.18 & 118.58 & 119.23 & 125.63 & 121.85 & 116.83 & 111.81 & 1.03 & 0.99 & 0.94 & \multirow[b]{2}{*}{ NO } \\
\hline & $\mathrm{EU}$ & 1696.86 & 1597.41 & 1660.21 & 1600.63 & 1589.36 & 1573.69 & 1511.29 & 1537.48 & 1546.94 & 1561.59 & 1493.56 & 1418.58 & 1343.52 & 0.97 & 0.92 & 0.87 & \\
\hline \multirow{2}{*}{$\begin{array}{l}\text { SDGI 07.11-Final energy } \\
\text { consumption (Million tons of oil } \\
\text { equivalent) }\end{array}$} & ES & 94.83 & 87.78 & 89.09 & 86.48 & 82.83 & 80.73 & 79.19 & 80.35 & 82.46 & 84.33 & 80.36 & 74.32 & 68.28 & 1.00 & 0.92 & 0.85 & \multirow[b]{2}{*}{ YES } \\
\hline & $\mathrm{EU}$ & 1180.27 & 1115.76 & 1163.13 & 1110.65 & 1110.62 & 1110.57 & 1065.57 & 1088.27 & 1110.08 & 1122.77 & 1085.27 & 1052.79 & 1020.27 & 1.00 & 0.97 & 0.94 & \\
\hline \multirow{2}{*}{$\begin{array}{l}\text { SDGI 07.30-Energy productivity } \\
\text { (Euro per kilogram of oil } \\
\text { equivalent) }\end{array}$} & ES & 7.40 & 7.80 & 7.80 & 7.70 & 7.50 & 8.00 & 8.20 & 8.20 & 8.40 & 8.30 & 8.65 & 9.14 & 9.64 & 1.05 & 1.11 & 1.18 & \multirow[b]{2}{*}{ NO } \\
\hline & $\mathrm{EU}$ & 7.10 & 7.20 & 7.10 & 7.40 & 7.50 & 7.60 & 8.00 & 8.10 & 8.20 & 8.30 & 8.78 & 9.53 & 10.27 & 1.08 & 1.18 & 1.27 & \\
\hline \multirow{2}{*}{$\begin{array}{l}\text { SDGI } 07.40-\text { Share of renewable } \\
\text { energy in gross final energy } \\
\text { consumption by sector }(\%)\end{array}$} & ES & 10.74 & 12.96 & 13.81 & 13.22 & 14.29 & 15.32 & 16.13 & 16.22 & 17.36 & 17.51 & 19.79 & 23.24 & 26.69 & 1.22 & 1.43 & 1.65 & \multirow[b]{2}{*}{ YES } \\
\hline & $\mathrm{EU}$ & 11.32 & 12.58 & 13.12 & 13.37 & 14.68 & 15.40 & 16.19 & 16.72 & 17.02 & 17.52 & 19.67 & 23.12 & 26.58 & 1.18 & 1.38 & 1.59 & \\
\hline
\end{tabular}

Source: Eurostat [50] and own estimations. 
Table 13. SDG 13: Take urgent action to combat climate change and its impacts.

\begin{tabular}{|c|c|c|c|c|c|c|c|c|c|c|c|c|c|c|c|c|c|c|}
\hline Indicators & & 2008 & 2009 & 2010 & 2011 & 2012 & 2013 & 2014 & 2015 & 2016 & 2017 & 2020 & 2025 & 2030 & I2020 & I2025 & I2030 & CONF. \\
\hline \multirow{2}{*}{$\begin{array}{l}\text { SDGI 13.10-Greenhouse gas } \\
\text { emissions (Source: European } \\
\text { Environment Agency) } \\
\text { (Greenhouse gas emissions [in } \\
\mathrm{CO}_{2} \text { equivalent], base year 1990) }\end{array}$} & ES & 144.50 & 130.80 & 125.90 & 126.20 & 123.80 & 114.60 & 115.60 & 119.70 & 116.40 & - & 104.48 & 89.64 & 74.78 & 0.87 & 0.75 & 0.62 & \multirow[b]{2}{*}{ NO } \\
\hline & $\mathrm{EU}$ & 90.60 & 84.00 & 85.80 & 83.10 & 82.00 & 80.40 & 77.40 & 78.00 & 77.60 & - & 70.74 & 63.27 & 55.78 & 0.91 & 0.81 & 0.72 & \\
\hline $\begin{array}{l}\text { SDGI 13.20-Greenhouse gas } \\
\text { emissions intensity of energy } \\
\text { consumption (source: EEA and } \\
\text { Eurostat) - index }(2000=100)\end{array}$ & EU & 94.90 & 93.70 & 92.50 & 92.00 & 91.80 & 90.50 & 89.10 & 88.70 & 87.50 & - & 84.20 & 79.79 & 75.37 & 0.95 & 0.90 & 0.85 & YES \\
\hline \multirow{2}{*}{$\begin{array}{l}\text { SDGI 13.50-Contribution to the } \\
\text { international 10obn USD } \\
\text { commitment on climate related } \\
\text { expending (Source: European } \\
\text { Environment Information and } \\
\text { Observation Network, European } \\
\text { Commission - Directorate-General } \\
\text { for Climate Action) (million EUR } \\
\text { [current prices]) }\end{array}$} & ES & - & - & - & - & - & - & 498.75 & 466.72 & 595.03 & 529.06 & $\begin{array}{ll}618.88 \\
\end{array}$ & 718.96 & 819.14 & 1.33 & 1.54 & 1.76 & \multirow[b]{2}{*}{ NO } \\
\hline & EU & - & - & - & - & - & - & $11,715.30$ & $13,813.88$ & $15,501.53$ & $14,924.63$ & $18,630.07$ & $24,021.81$ & $29,419.46$ & 1.35 & 1.74 & 2.13 & \\
\hline \multirow{2}{*}{$\begin{array}{l}\text { SDGI 07.10-Primary energy } \\
\text { consumption (Million tons of oil } \\
\text { equivalent) }\end{array}$} & ES & 134.44 & 123.37 & 123.32 & 122.96 & 123.39 & 116.05 & 114.18 & 118.58 & 119.23 & 125.63 & 121.85 & 116.83 & 111.81 & 1.03 & 0.99 & 0.94 & \multirow[b]{2}{*}{ NO } \\
\hline & EU & 1696.86 & 1597.41 & 1660.21 & 1600.63 & 1589.36 & 1573.69 & 1511.29 & 1537.48 & 1546.94 & 1561.59 & 1493.56 & 1418.58 & 1343.52 & 0.97 & 0.92 & 0.87 & \\
\hline \multirow{2}{*}{$\begin{array}{l}\text { SDGI 07.11-Final energy } \\
\text { consumption (Million tons of oil } \\
\text { equivalent) }\end{array}$} & ES & 94.83 & 87.78 & 89.09 & 86.48 & 82.83 & 80.73 & 79.19 & 80.35 & 82.46 & 84.33 & 80.36 & 74.32 & 68.28 & 1.00 & 0.92 & 0.85 & \multirow[b]{2}{*}{ YES } \\
\hline & $\mathrm{EU}$ & 1180.27 & 1115.76 & 1163.13 & 1110.65 & 1110.62 & 1110.57 & 1065.57 & 1088.27 & 1110.08 & 1122.77 & 1085.27 & 1052.79 & 1020.27 & 1.00 & 0.97 & 0.94 & \\
\hline $\begin{array}{l}\text { SDGI 07.40-Share of renewable } \\
\text { energy in gross final energy } \\
\text { consumption by sector }(\%)\end{array}$ & ES & 10.74 & 12.96 & 13.81 & 13.22 & 14.29 & 15.32 & 16.13 & 16.22 & 17.36 & 17.51 & 19.79 & 23.24 & 26.69 & 1.22 & 1.43 & 1.65 & YES \\
\hline \multirow{2}{*}{$\begin{array}{l}\text { SDGI 12.30-Average } \mathrm{CO}_{2} \\
\text { emissions per km from new } \\
\text { passenger cars (Source: European } \\
\text { Environment Agency, European } \\
\text { Commission-Directorate-General } \\
\text { for Climate Action) }\left(\mathrm{g} \mathrm{CO}_{2} \text { per }\right. \\
\mathrm{km} \text { ) }\end{array}$} & ES & 148.20 & 142.20 & 137.90 & 133.80 & 128.70 & 122.40 & 118.60 & 115.30 & 114.40 & 115.00 & 116.28 & 118.42 & 120.56 & 1.01 & 1.03 & 1.05 & \multirow[t]{2}{*}{ NO } \\
\hline & EU & - & - & - & - & - & - & 123.40 & 119.50 & 118.10 & 118.50 & 113.35 & 105.66 & 97.96 & 0.95 & 0.88 & 0.82 & \\
\hline
\end{tabular}

Source: Eurostat [50] and own estimations. 
Table 14. SDG 14: Conserve and sustainably use the oceans, seas and marine resources for sustainable development.

\begin{tabular}{|c|c|c|c|c|c|c|c|c|c|c|c|c|c|c|c|c|c|c|}
\hline Indicators & & 2008 & 2009 & 2010 & 2011 & 2012 & 2013 & 2014 & 2015 & 2016 & 2017 & 2020 & 2025 & 2030 & I2020 & I2025 & I2030 & CONF. \\
\hline \multirow{2}{*}{$\begin{array}{l}\text { SDGI 14.10-Surface of marine } \\
\text { sites designated under NATURA } \\
2000 \text { (Source: European } \\
\text { Environment Agency, European } \\
\text { Commission-Directorate-General } \\
\text { for Environment) }\left(\mathrm{km}^{2}\right)\end{array}$} & ES & - & - & - & 10,193 & 10,193 & 10,637 & 71,677 & 84,386 & 84,404 & 84,404 & 152,778 & 231,922 & 311,153 & 1.81 & 2.75 & 3.69 & \multirow{2}{*}{ NO } \\
\hline & $\mathrm{EU}$ & - & - & - & - & - & 251,566 & 318,127 & 360,350 & 395,528 & 532,417 & 709,634 & $1,036,635$ & $1,363,995$ & 1.97 & 2.88 & 3.79 & \\
\hline \multirow{2}{*}{$\begin{array}{l}\text { SDGI } 14.40 \text {-Bathing sites with } \\
\text { excellent water quality by locality } \\
\text { (Source: European Environment } \\
\text { Agency) }\end{array}$} & ES & - & - & - & 1927 & 1926 & 1931 & 1942 & 1948 & 1949 & 1960 & 1976 & 2005 & 2033 & 1.01 & 1.03 & 1.04 & \multirow[t]{2}{*}{ YES } \\
\hline & EU & - & - & - & 15,444 & 15,406 & 15,436 & 14,828 & 14,791 & 14,821 & 14,935 & 14,370 & 13,774 & 13,177 & 0.97 & 0.93 & 0.89 & \\
\hline
\end{tabular}

Source: Eurostat [50] and own estimations.

Table 15. SDG 15: Protect, restore and promote sustainable use of terrestrial ecosystems, sustainably manage forests, combat desertification, and halt and reverse land degradation and halt biodiversity loss.

\begin{tabular}{|c|c|c|c|c|c|c|c|c|c|c|c|c|c|c|c|c|c|c|}
\hline Indicators & & 2008 & 2009 & 2010 & 2011 & 2012 & 2013 & 2014 & 2015 & 2016 & 2017 & 2020 & 2025 & 2030 & I2020 & I2025 & I2030 & CONF. \\
\hline $\begin{array}{l}\text { SDGI 15.20-Surface of terrestrial } \\
\text { sites designated under NATURA }\end{array}$ & ES & - & - & - & 137,510 & 137,664 & 137,365 & 137,444 & 137,757 & 137,872 & 137,952 & 138,142 & 138,521 & 138,900 & 1.00 & 1.01 & 1.01 & \multirow{2}{*}{ YES } \\
\hline $\begin{array}{l}2000 \text { (Source: European } \\
\text { Environment Agency, European } \\
\text { Commission-Directorate-General } \\
\text { for Environment) }\left(\mathrm{km}^{2}\right)\end{array}$ & EU & - & - & - & - & - & 787,766 & 788,488 & 787,606 & 789,081 & 790,213 & 791,807 & 794,812 & 797,820 & 1.01 & 1.01 & 1.01 & \\
\hline $\begin{array}{l}\text { SDGI 06.40-Nitrate in } \\
\text { groundwater (Source: European }\end{array}$ & ES & 40.20 & 37.40 & 37.20 & 38.10 & 39.50 & 39.50 & 39.50 & 39.50 & - & - & 40.34 & 41.15 & 41.97 & 1.02 & 1.04 & 1.06 & \multirow{2}{*}{$\mathrm{NO}$} \\
\hline $\begin{array}{l}\text { Environment Agency) (mg NO} \\
\text { per liter) }\end{array}$ & EU & 18.90 & 18.40 & 18.80 & 18.30 & 18.40 & 18.60 & 18.30 & 18.30 & - & - & 18.07 & 18.00 & 17.33 & 0.99 & 0.98 & 0.95 & \\
\hline
\end{tabular}


Table 16. SDG 16: Promote peaceful and inclusive societies for sustainable development, provide access to justice for all and build effective, accountable and inclusive institutions at all levels.

\begin{tabular}{|c|c|c|c|c|c|c|c|c|c|c|c|c|c|c|c|c|c|c|}
\hline Indicators & & 2008 & 2009 & 2010 & 2011 & 2012 & 2013 & 2014 & 2015 & 2016 & 2017 & 2020 & 2025 & 2030 & I2020 & I2025 & I2030 & CONF. \\
\hline \multirow{2}{*}{$\begin{array}{l}\text { SDGI 16.10—Death rate due to } \\
\text { homicide by sex (number par } \\
\text { 100,000 persons) }\end{array}$} & ES & 0.80 & 0.70 & 0.70 & 0.68 & 0.62 & 0.65 & 0.64 & 0.58 & - & - & 0.53 & 0.40 & 0.27 & 0.92 & 0.70 & 0.46 & \multirow{2}{*}{ NO } \\
\hline & EU & 1.00 & 0.90 & 0.90 & 0.85 & 0.81 & 0.77 & 0.70 & 0.69 & - & - & 0.48 & 0.26 & 0.02 & 0.69 & 0.38 & 0.03 & \\
\hline \multirow{2}{*}{$\begin{array}{l}\text { SDGI 16.20—Population } \\
\text { reporting occurrence of crime, } \\
\text { violence or vandalism in their } \\
\text { area by poverty status (\%) }\end{array}$} & ES & 14.70 & 16.40 & 13.00 & 10.80 & 10.10 & 14.20 & 11.90 & 10.00 & 10.30 & 8.70 & 7.13 & 3.98 & 0.82 & 0.71 & 0.40 & 0.08 & \multirow[b]{2}{*}{ YES } \\
\hline & EU & - & - & 14.40 & 14.10 & 13.60 & 14.50 & 14.00 & 13.60 & 13.00 & 12.00 & 11.70 & 10.35 & 9.00 & 0.86 & 0.76 & 0.66 & \\
\hline \multirow{2}{*}{$\begin{array}{l}\text { SDGI 16.30-General government } \\
\text { total expenditure on law courts } \\
\text { (Million euro) }\end{array}$} & ES & 3826 & 4129 & 4194 & 4088 & 3862 & 3759 & 3699 & 3877 & 3875 & 3993 & 3923.13 & 3830.12 & 3737.01 & 1.01 & 0.99 & 0.96 & \multirow{2}{*}{ NO } \\
\hline & EU & 46,146 & 46,218 & 47,315 & 48,533 & 48,381 & 48,487 & 49,272 & 50,020 & 50,147 & 50,975 & $52,462.97$ & $55,093.95$ & $57,727.82$ & 1.05 & 1.10 & 1.15 & \\
\hline \multirow{2}{*}{$\begin{array}{l}\text { SDGI 16.60-Population with } \\
\text { confidence in EU institutions by } \\
\text { institution (Source: European } \\
\text { Commission-Directorate-General } \\
\text { for Communication) (\%) }\end{array}$} & ES & 57 & 53 & 39 & 33 & 25 & 23 & 28 & 27 & 33 & 41 & 34.44 & 23.50 & 12.56 & 1.28 & 0.87 & 0.47 & \multirow[b]{2}{*}{ NO } \\
\hline & EU & 51 & 50 & 48 & 41 & 44 & 39 & 42 & 38 & 42 & 45 & 42.05 & 37.15 & 32.24 & 1.11 & 0.98 & 0.85 & \\
\hline
\end{tabular}

Source: Eurostat [50] and own estimations. 
Table 17. SDG 17: Strengthen the means of implementation and revitalize the Global Partnership for Sustainable Development.

\begin{tabular}{|c|c|c|c|c|c|c|c|c|c|c|c|c|c|c|c|c|c|c|}
\hline Indicators & & 2008 & 2009 & 2010 & 2011 & 2012 & 2013 & 2014 & 2015 & 2016 & 2017 & 2020 & 2025 & 2030 & I2020 & I2025 & I2030 & CONF. \\
\hline \multirow{2}{*}{$\begin{array}{l}\text { SDGI 17.10-Official } \\
\text { development assistance as share } \\
\text { of gross national income (Source: } \\
\text { Organization for Economic } \\
\text { Co-operation and Development) } \\
\text { (\% of gross national income)) }\end{array}$} & ES & 0.45 & 0.46 & 0.43 & 0.29 & 0.16 & 0.17 & 0.13 & 0.12 & 0.34 & 0.19 & 0.11 & -0.05 & -0.21 & 0.91 & -0.40 & -1.71 & \multirow[b]{2}{*}{ NO } \\
\hline & EU & 0.40 & 0.42 & 0.44 & 0.42 & 0.39 & 0.41 & 0.41 & 0.46 & 0.53 & 0.50 & 0.54 & 0.59 & 0.64 & 1.16 & 1.28 & 1.40 & \\
\hline \multirow{2}{*}{$\begin{array}{l}\text { SDGI 17.20-EU financing to } \\
\text { developing countries by financing } \\
\text { source (Source: Organization for } \\
\text { Economic Co-operation and } \\
\text { Development) (million EUR } \\
\text { [current prices]) }\end{array}$} & ES & 20,456 & 9186 & 7800 & 14,472 & 1539 & 6014 & 10,289 & 19,866 & 4258 & 2301 & 8148.15 & -733.75 & -999.80 & 0.41 & -0.04 & -0.05 & \multirow[b]{2}{*}{ NO } \\
\hline & EU & 119,718 & 102,203 & 127,336 & 153,784 & 147,962 & 129,811 & 171,987 & 178,101 & 143,007 & 155,224 & 189,764 & 196,918 & 225,576 & 1.07 & 1.11 & 1.27 & \\
\hline \multirow{2}{*}{$\begin{array}{l}\text { SDGI 17.30—EU imports from } \\
\text { developing countries by country } \\
\text { income groups (million EUR) }\end{array}$} & ES & 76,616 & 51,928 & 66,735 & 75,388 & 80,650 & 77,020 & 79,482 & 77,589 & 74,762 & 88,378 & $90,201.68$ & $99,955.87$ & $109,720.75$ & 1.16 & 1.29 & 1.41 & \multirow[b]{2}{*}{ NO } \\
\hline & $\mathrm{EU}$ & 762,471 & 591,995 & 756,791 & 841,518 & 863,783 & 817,475 & 838,537 & 881,805 & 869,710 & 957,870 & $1,013,951$ & $1,142,207$ & $1,270,603$ & 1.15 & 1.30 & 1.44 & \\
\hline \multirow{2}{*}{$\begin{array}{l}\text { SDGI } 17.40-\text { General government } \\
\text { gross debt } \% \text { of gross domestic } \\
\text { product) }\end{array}$} & ES & 39.50 & 52.80 & 60.10 & 69.50 & 85.70 & 95.50 & 100.40 & 99.30 & 99.00 & 98.10 & 95.58 & 91.37 & 87.16 & 0.96 & 0.92 & 0.88 & \multirow[b]{2}{*}{ YES } \\
\hline & EU & 60.70 & 73.30 & 79.00 & 81.60 & 84.00 & 85.80 & 86.60 & 84.60 & 83.40 & 81.70 & 87.24 & 96.46 & 105.69 & 1.03 & 1.14 & 1.25 & \\
\hline \multirow{2}{*}{$\begin{array}{l}\text { SDGI 17.50-Shares of } \\
\text { environmental and labor taxes in } \\
\text { total tax revenues (\% of total } \\
\text { taxes) }\end{array}$} & ES & 5.06 & 5.39 & 5.22 & 5.05 & 4.88 & 5.80 & 5.55 & 5.73 & 5.56 & 5.43 & 5.75 & 6.04 & 6.33 & 1.00 & 1.05 & 1.11 & \multirow{2}{*}{ YES } \\
\hline & EU & 6.03 & 6.34 & 6.37 & 6.37 & 6.35 & 6.33 & 6.33 & 6.31 & 6.30 & 6.14 & 6.16 & 6.17 & 6.18 & 0.98 & 0.98 & 0.98 & \\
\hline
\end{tabular}

Source: Eurostat [50] and own estimations. 
In order to calculate the estimated value for each indicator in years 2020, 2025, and 2030, both in Spain an in the whole EU, we use the FORECAST.ETS function at Microsoft Office 365 Excel software. Using the additive error, additive trend, and additive seasonality version of the Exponential Triple Smoothing algorithm (ETS AAA), this function predicts a future value, based on historical time-based data. It is a progression upon the array of historical known values to the target date, on a continuing time-line [2,51-53]. This technique allows for assessing whether estimated values for each indicator at Spain level will be near and along with the EU average values for the analyzed time-period.

The technique of dynamic indices enables a comparison or establishes a relationship among the data obtained for different periods in a temporal series regarding a single indicator measuring homogeneous facts $[2,54,55]$. In this paper, we start from the 2015 value for each indicator as the base to check forecasted values in years 2020, 2025, and 2030. Year 2015 as starting point is relevant because it was back then when the 2030 Agenda was agreed and States initiated into the process to achieve SDG.

The main findings found and derived from the analysis presented above in Tables 1-17 for Spain are summarized below.

Out of the data provided in Table 1, containing indicators useful to diagnose confluence in SDG 01, end poverty in all its forms everywhere, we anticipate that Spain will be capable of achieving, in the year 2030, the European average values for indicators concerning dwelling conditions (SDGI 01.60 and SDGI 06.10), self-reported unmet need for medical examination and care (SDGI 03.60) and adaptation of housing size to family units (SDGI 11.10). However, there is a critical gap with EU reference regarding poverty and social exclusion (SDGI 01.10 and SDGI 01.20), situations of material deprivation and difficulties to properly warm homes (SDGI 01.30 and SDGI 07.60) or to get a decent, long-term, accurately paid job (SDGI 01.40 and SDGI 01.41).

As for SDG 02, end hunger, achieve food security and improved nutrition and promote sustainable agriculture, studied in Table 2, Spain will be able to reach the European average values in 2030 only for indicators related to agricultural working productivity (SDGI 02.20) and area under organic farming (SDGI 02.40). Instead, there will be no junction with EU in government support to agricultural research and development (SDGI 02.30), gross nutrient balance on agricultural land by nutrient (SDGI 02.50), ammonia emissions from agriculture (SDGI 02.60) or nitrate in groundwater (SDGI 06.40).

Nonetheless, a considerable level of confluence between Spain and EU can be appreciated regarding indicators for SDG 03, ensure healthy lives and promote well-being for all at all ages. Actually, Spain gets better prognoses than EU regarding life expectancy at birth (SDGI 03.10), perceived health (SDGI 03.20), death rate due to chronic diseases or to tuberculosis, HIV and hepatitis (SDGI 03.40 y SDGI 03.41), self-reported unmet need for medical examination and care (SDGI 03.60), death rate due to at-work or road accidents (SDGI 08.60 and SDGI 11.40) and acoustic pollution (SDGI 11.20). Only air pollution predicted values leave Spain behind the average values for EU (SDGI 11.50).

In SDG 04, ensure inclusive and equitable quality education and promote lifelong learning opportunities for all, evolution foreseen in 5 of 6 indicators is not auspicious for Spain (Table 4). The only indicator in which the forecast is positive is the rate of early leavers from education and training (SDGI 04.10). Subsequently, we may conclude that Spain would not reach the reference average EU rates regarding population with tertiary educational attainment (SDGI 04.20), early childhood education (SDGI 04.30), recent graduates employment rates (SDGI 04.50), adult participation in learning (SDGI 04.60), and proportion of young people unemployed and not engaged in education or in training programs (SDGI 08.20).

In contrast, Spanish situation regarding SDG 05, achieve gender equality and empower all women and girls, is very promising as 5 out of 8 indicators reach the EU reference values for 2030 (Table 5). Specifically, alignment is obtained in relation to the reduction of unfair gender pay gap (SDGI 05.20 y SDGI 05.30), inactive population due to caring responsibilities (SDGI 05.40), presence of women in national parliaments and governments (SDGI 05.50) and early leavers from education and training (SDGI 04.10). Yet, situation is adverse in aspects such as the presence of women in senior 
management positions (SDGI 05.60), tertiary educational attainment (SDGI 04.20) and employment of recent graduates (SDGI 04.50).

Indicators related to the follow up of SDG 06 (Table 6), ensure availability and sustainable management of water and sanitation for all, provide positive outlooks for Spain comparing to EU regarding access to sanitary facilities in households (SDGI 06.10) and bathing sites with excellent water quality (SDGI 14.40). Nevertheless, the situation is unfavorable regarding nitrate in groundwater, as Spanish foresights are quite higher than EU ones for year 2030 (SDGI 06.40).

Considering SDG 07 (Table 7), ensure access to affordable, reliable, sustainable and modern energy for all, Spain presents a positive trend regarding European average rates for indicators considering final and per capita energy consumption (SDGI 07.11 and SDGI 07.20), renewable energy consumption (SDGI 07.40) and greenhouse gas emission intensity of energy consumption (SDGI 13.20). Still, within the current framework, confluence would not be possible in the fields of primary energy consumption (SDGI 07.10), energy productivity (SDGI 07.30), energy import dependency (SDGI 07.50) and population unable to keep home adequately warm (SDGI 07.60).

Out of data presented in Table 8, useful to assess confluence in SDG 08, promote sustained, inclusive and sustainable economic growth, full and productive employment and decent work for all, Spain would be able to reach reference EU values in 2030 only regarding the rates of people killed in accidents at work (SDGI 08.60), inactive population due to caring responsibilities (SDGI 05.40) and resource productivity and domestic material consumption (SDGI 12.20). Yet, estimations show that the gap with Europe will persist in aspects as Gross Domestic Product (GDP) per capita (SDGI 08.10), investment share of GDP by institutional sector (SDGI 08.11), proportion of young people unemployed and not engaged in education or in training programs (SDGI 08.20), long-term unemployment rate (SDGI 08.40) and in work at risk of poverty rate (SDGI 01.41).

For SDG 09, build resilient infrastructure, promote inclusive and sustainable industrialization and foster innovation, indicators calculated in Table 9 indicate that Spain will be in position to improve the rates foreseen for EU only regarding the number of patent applications (SDGI 09.40). Confluence with average European values for 2030 is not achieved in all the remainder issues: gross domestic expenditure on research and development (R\&D) (SDGI 09.10), employment in high- and medium-high technology manufacturing and knowledge-intensive services (SDGI 09.20), R\&D personnel (SDGI 09.30), use of busses and trains (SDGI 09.50), share of rail and inland waterways in total freight transport (SDGI 09.60), and average carbon dioxide emissions from new passenger cars (SDGI 12.30).

Spain is in a really bad position regarding SDG10, reduce inequality within and among countries, as no indicator achieves the reference average EU values for 2030 (Table 10). There are severe sub-par differences regarding purchasing power (SDGI 10.10), adjusted gross disposable income of households (SDGI 10.20), at risk of poverty gap (SDGI 10.30), income distribution (SDGI 10.41), income share of the poorest population (SDGI 10.50), rate of asylum applications (SDGI 10.60), financing to developing countries (SDGI 17.20) and rate of imports from developing countries (SDGI 17.30). Every item related to public investment probably has suffered the consequences of 2008-2014 recession.

Indicators used to monitor SDG 11 (Table 11), make cities and human settlements inclusive, safe, resilient and sustainable, let us observe a positive trend in Spain comparing to EU regarding adaptation of housing size to family units (SDGI 11.10), noise pollution (SDGI 11.20), people killed in road accidents (SDGI 11.40), habitability conditions (SDGI 01.60) and population reporting occurrence of crime, violence or vandalism in their area (SDGI 16.20). Issues needed for improvement and with a detrimental side are exposure to air pollution by particulate matter (SDGI 11.50), recycling rate of municipal waste (SDGI 11.60) and rates of share of busses and trains (SDGI 09.50).

As for SDG 12 (Table 12), ensure sustainable consumption and production patterns, Spain achieves European reference values only for indicators involved in resource productivity (SDGI 12.20), evolution in final energy consumption (SDGI 07.11) and renewable energy consumption (SDGI 07.40). However, provided the ongoing trend, alignment with Europe will not be possible in issues regarding average carbon dioxide emissions from new passenger cars (SDGI 12.30), circular material use rate (SDGI 
12.41), evolution in primary energy consumption rates (SDGI 07.10) and energy productivity (SDGI 07.30). That might be the reason why the Spanish acting government has recently exhibited a strong commitment to change the production pattern and undertake a profound ecological transition of economy [11].

In SDG 13, take urgent action to combat climate change and its impacts, values obtained in 4 out of 7 indicators provide a better situation for Spain than for EU in 2030 regarding greenhouse gas emissions intensity of energy consumption (SDGI 13.20), final energy consumption (SDGI 07.11) and renewable energy consumption (SDGI 7.40). On the contrary, forecasts are unfavorable for Spain in comparison to Europe in 2030 regarding greenhouse gas emissions (SDGI 13.10), commitment on climate related expending (SDGI 13.50), primary energy consumption (SDGI 07.10) and average carbon dioxide emissions from new passenger cars (SDGI 12.30).

To study SDG 14 (Table 14), conserve and sustainably use the oceans, seas and marine resources for sustainable development, and SDG 15 (Table 15), protect, restore and promote sustainable use of terrestrial ecosystems, sustainably manage forests, combat desertification, and halt and reverse land degradation and halt biodiversity loss, there is information available only for a pair of indicators in each goal. Out of them, Spain presents better prognoses than the EU at the horizon of 2030 for bathing sites with excellent water quality (SDGI 14.40); similar values regarding surface of terrestrial sites designated under NATURA 2000 (SDGI 15.20); slightly sub-par merits concerning surface of marine sites designated under NATURA 2000 (SDGI 14.10) and a major increase on levels of nitrate in groundwater (SDGI 06.40).

In Table 16, we can find items to screen eventual confluence in SDG 16, promote peaceful and inclusive societies for sustainable development, provide access to justice for all and build effective, accountable and inclusive institutions at all levels. The forecast shows that Spain will be able to reach EU average only regarding population reporting occurrence of crime, violence or vandalism in their area (SDGI 16.20). Instead, a relevant gap will persist for death rate due to homicide (SDGI 16.10), general government total expenditure on law courts (SDGI 16.30) and population with confidence in EU institutions (SDGI 16.60).

Finally, for SDG 17 (Table 17), strengthen the means of implementation and revitalize the Global Partnership for Sustainable Development, predictions are encouraging for Spain regarding general government gross debt (SDGI 17.40) and shares of environmental and labor taxes in total tax revenues (SDGI 17.50). On the contrary, out of the evolution of indicators tied to official development assistance as share of gross national income (SDGI 17.10), financing to developing countries (SDGI 17.20) and imports from developing countries (SDGI 17.30) Spain will not reach EU average values for year 2030.

In brief, and as it can be seen in Table 18, presenting confluence rate for each SDG, Spain must definitely take measures to revert the current trend and try to achieve better results in each and every area of 2030 Agenda. In particular, indicators reaching the EU average values at the horizon of 2030 are less than 50\% in 10 goals (SDG 01, SDG 02, SDG 04, SDG 08, SDG 09, SDG 10, SDG 12, SDG 13, SDG 16 y SDG 17), equal or over $50 \%$ but under 75\% in 5 goals (SDG 05, SDG 06, SDG 07, SDG 11, SDG 14 y SDG 15) and over 75\% only in one (SDG 03). 
Table 18. Spain/EU confluence rate for SDG.

\begin{tabular}{|c|c|c|c|c|}
\hline Sustainable Development Goals & $\begin{array}{l}\text { Number of } \\
\text { Indicators } \\
\text { Achieving EU } \\
\text { Reference Values } \\
\text { for Year } 2030\end{array}$ & $\begin{array}{l}\text { Number of } \\
\text { Indicators not } \\
\text { Achieving EU } \\
\text { Reference Values } \\
\text { for Year } 2030\end{array}$ & $\begin{array}{l}\text { Total Number of } \\
\text { Analyzed } \\
\text { Indicators for } \\
\text { Each Goal }\end{array}$ & $\begin{array}{l}\text { Percentage of } \\
\text { Confluence }\end{array}$ \\
\hline $\begin{array}{l}\text { 01. End poverty in all its forms } \\
\text { everywhere. }\end{array}$ & 4 & 6 & 10 & 40.00 \\
\hline $\begin{array}{l}\text { 02. End hunger, achieve food security } \\
\text { and improved nutrition and promote } \\
\text { sustainable agriculture. }\end{array}$ & 2 & 4 & 6 & 33.33 \\
\hline $\begin{array}{l}\text { 03. Ensure healthy lives and promote } \\
\text { well-being for all at all ages. }\end{array}$ & 8 & 1 & 9 & 88.89 \\
\hline $\begin{array}{l}\text { 04. Ensure inclusive and equitable quality } \\
\text { education and promote lifelong learning } \\
\text { opportunities for all. }\end{array}$ & 1 & 5 & 6 & 16.67 \\
\hline $\begin{array}{l}\text { 05. Achieve gender equality and } \\
\text { empower all women and girls. }\end{array}$ & 5 & 3 & 8 & 62.50 \\
\hline $\begin{array}{l}\text { 06. Ensure availability and sustainable } \\
\text { management of water and sanitation for } \\
\text { all. }\end{array}$ & 2 & 1 & 3 & 66.67 \\
\hline $\begin{array}{l}\text { 07. Ensure access to affordable, reliable, } \\
\text { sustainable and modern energy for all. }\end{array}$ & 4 & 4 & 8 & 50.00 \\
\hline $\begin{array}{l}\text { 08. Promote sustained, inclusive and } \\
\text { sustainable economic growth, full and } \\
\text { productive employment and decent work } \\
\text { for all. }\end{array}$ & 3 & 6 & 9 & 33.33 \\
\hline $\begin{array}{l}\text { 09. Build resilient infrastructure, promote } \\
\text { inclusive and sustainable } \\
\text { industrialization and foster innovation. }\end{array}$ & 1 & 6 & 7 & 14.29 \\
\hline $\begin{array}{l}\text { 10. Reduce inequality within and among } \\
\text { countries. }\end{array}$ & 0 & 8 & 8 & 0.00 \\
\hline $\begin{array}{l}\text { 11. Make cities and human settlements } \\
\text { inclusive, safe, resilient and sustainable. }\end{array}$ & 5 & 3 & 8 & 62.50 \\
\hline $\begin{array}{l}\text { 12. Ensure sustainable consumption and } \\
\text { production patterns. }\end{array}$ & 3 & 4 & 7 & 42.86 \\
\hline $\begin{array}{l}\text { 13. Take urgent action to combat climate } \\
\text { change and its impacts. }\end{array}$ & 3 & 4 & 7 & 42.86 \\
\hline $\begin{array}{l}\text { 14. Conserve and sustainably use the } \\
\text { oceans, seas and marine resources for } \\
\text { sustainable development. }\end{array}$ & 1 & 1 & 2 & 50.00 \\
\hline $\begin{array}{l}\text { 15. Protect, restore and promote } \\
\text { sustainable use of terrestrial ecosystems, } \\
\text { sustainably manage forests, combat } \\
\text { desertification, and halt and reverse land } \\
\text { degradation and halt biodiversity loss. }\end{array}$ & 1 & 1 & 2 & 50.00 \\
\hline $\begin{array}{l}\text { 16. Promote peaceful and inclusive } \\
\text { societies for sustainable development, } \\
\text { provide access to justice for all and build } \\
\text { effective, accountable and inclusive } \\
\text { institutions at all levels. }\end{array}$ & 1 & 3 & 4 & 25.00 \\
\hline $\begin{array}{l}\text { 17. Strengthen the means of } \\
\text { implementation and revitalize the Global } \\
\text { Partnership for Sustainable Development. }\end{array}$ & 2 & 3 & 5 & 40.00 \\
\hline TOTAL & 46 & 63 & 109 & 42.20 \\
\hline
\end{tabular}

Table 19 presents the different indicators previously used now grouped into three categories, according to the economic, environment or social dimension of the indicators. The aim of this classification is to assess the degree of Spain confluence with the EU for each dimension separately. It allows concluding that projections for the economic and environmental dimensions are quite adverse ( $28.57 \%$ y $35.71 \%$ respectively), while the perspective is comparatively better in everything related to social aspects as confluence is there reached in almost half of the indicators $(47.06 \%)$. 
Table 19. Spain/EU confluence rate by categories.

\begin{tabular}{ccccc}
\hline Categories & $\begin{array}{c}\text { Number of } \\
\text { Indicators } \\
\text { Achieving EU } \\
\text { Reference Values } \\
\text { for Year 2030 }\end{array}$ & $\begin{array}{c}\text { Number of } \\
\text { Indicators not } \\
\text { Achieving EU } \\
\text { Reference Values } \\
\text { for Year 2030 }\end{array}$ & $\begin{array}{c}\text { Total Number of } \\
\text { Analyzed } \\
\text { Indicators for } \\
\text { Category }\end{array}$ & $\begin{array}{c}\text { Percentage of } \\
\text { Confluence }\end{array}$ \\
\hline Economic & 8 & 20 & 28 & 28.57 \\
Environment & 5 & 9 & 14 & 35.71 \\
Social & 16 & 18 & 34 & 47.06 \\
\hline TOTAL & 29 & 47 & 76 & 38.16 \\
\hline \multicolumn{5}{c}{ Source: Own production. }
\end{tabular}

In order to put the results in context, it is interesting to make a comparison with the data available in other countries for which similar studies have been carried out, such as Romania [2] and Poland [1] (see Table 20). In Romania case [2] there is a national research that use the information provided by Eurostat, and that is based on a methodology similar to the one that was here used for Spain; a homogeneous comparison of the results can be therefore displayed. Nevertheless, research involving Poland [1] has instead used national indicators, provided by their National Statistics Office, and although the methodology is also based upon dynamic indexes, no forecasting tools for 2030 are applied, nor comparisons with EU average rate are developed.

Table 20. Compliance with 2030 Agenda in Poland, Romania, and Spain.

\begin{tabular}{cccc}
\hline Categories & Poland & Romania & Spain \\
\hline Economic & 75.00 & 40.00 & 28.57 \\
Environment & 75.00 & 56.25 & 35.71 \\
Social & 81.82 & 28.57 & 47.06 \\
\hline TOTAL & 78.08 & 38.16 & 38.16 \\
\hline \multicolumn{4}{c}{ Source: Own production. }
\end{tabular}

Dividing the results of each research among the three aforementioned categories, Poland is the best-placed country to fulfill the 2030 commitments. Poland progresses towards sustainable development by reducing social inequalities, promoting environmental care and ensuring a proper quality of life.

On the other hand, Romania a Spain have a long road ahead and must undertake reforms and implement policies to reduce the gap with the EU. Romania is in a positive condition regarding environmental aspects, but needs to work in economic and, specially, in social issues. Rather, Spain is in a positive situation in social dimension but is clearly at a drawback in the environmental and economic aspects of Agenda 2030.

\section{Discussion}

The results obtained in this work have serious managerial implications if Spain wants to meet the SDG commitments linked to Agenda 2030. Spain must, immediately, take measures and promote reforms to change the trend with regard to the problems identified. The following are the main areas that we believe require special attention.

For instance, in order to accomplish the goal of ending poverty in all its forms, the Finnish example could be inspiring [56]. Despite the very different national context, a universal basic income combined with programs promoting access to accommodation, popular also in other European countries [57], may be useful in order to advance towards poverty and social exclusion eradication. Parallel to that, Spain could also experiment on tax incentives $[58,59]$ with the objective of stimulating job offers to the most vulnerable groups because, as the European Training Foundation has remarked, the inclusive 
growth and job creation agenda entails clear linkages to skills development and social inclusion policies. An agreement exists that making growth more inclusive would also make it more sustainable [60].

Agriculture activities should be more concerned about environmental challenges. The amount of nitrate in groundwater, the abuse of nutrients and ammonia emissions must become focal points for a change. Spain should lay the foundations for a sustainable agriculture, proficient enough to foster the transition to organic farming activities, capable of generating added value for consumers and reducing environmental pollution. Spain seems to have already good conditions for the development of this type of agriculture due to its favorable climatology and the extensive production systems traditionally applied in a large number of crops [61], but according to the study carried out in this paper, there is still a long path ahead.

As for education, it is necessary to work on quality. Measures to expand the network of early schooling and the design of complementary support measures, at all stages of training, might have a positive impact to avoid dropping out of school. Tax incentives for the recruitment of new graduates would increase the added value of University training. Citizenship education needs to be implemented as well in adult population.

Although Spain obtains a good result in gender equality policies, measures to promote and actively regulate the presence of women in decision-making and management positions in companies are still needed [62].

It is vital to go ahead promoting sustainable energy generation using renewable sources, which will make possible to avoid dependence on energy from other countries. Favoring self-generation and self-consumption in clean energy households, through autonomous energy production systems is as well crucial. Not only is that a goal included in 2030 Agenda, but also a shorter-term objective expressly assumed by Spain in a EU context [63].

Spanish economy is highly conditioned by an extremely high unemployment rate [64]. It is a serious problem that generates inequalities, causes exclusion and affects social cohesion. The difficulty of accessing a decent and adequately paid work and the high rate of temporary contracts are factors that suggest a reform in the job market regulation which in consultation with the social partners, may introduce a social perspective into labor relationships.

A pending issue in Spain has to do with the investment in R\&D and the development of knowledge and information technologies. Although there have been formal government efforts to revert the situation [65], this paper proves that they have been till now insufficient. New measures need to be enhanced. Again, tax deductions could be implemented for companies making this sort of investments.

Social concern on sustainability has to be boosted [16]. It is advisable to launch social awareness campaigns to promote the recycling of materials, the use of public transports and vehicles with zero or low carbon emissions in order to reduce pollution. Taxes penalizing environmentally irresponsible behavior could be introduced to help the achievement of these goals.

In Spain there are major inequalities in terms of national wealth distribution. Without redistributive measures, inequalities could increase substantially and could lead to greater social conflict. Hence, the existing inequalities between Spanish regions have to be accounted and an investment plan for the most disadvantaged regions should be drawn up in order to attach population to land, ensure a certain social well-being and reduce the inequality gap [66]. The Spanish situation is somewhat similar to UN global appreciation [67] when it points out the slow-paced progress to many Sustainable Development Goals in recent years. Certainly, UN has also concluded that the most vulnerable people and countries are still suffering the most, and that the global reaction has not been determined enough.

Also regarding regional differences and provided past and more recent experiences in historical backgrounds such as Basque Country and Catalonia, an essential task for political action is to guarantee peaceful coexistence within the nation and to generate a framework of mutual trust. In the other hand it is urgent to strengthen public institutions, and especially the judiciary, promoting equal access to justice for all. 
Eventually, provided national economic recovery, it is necessary to retrieve the path of development aid policies to developing countries in order to fight humanitarian crises.

\section{Conclusions}

Summing up, Spain has a long way to go in order to fulfill commitment to the 2030 Agenda and meet EU standards. Such a diagnosis is confirmed by our calculations and strongly conditioned by the current situation of both political and social crises in the country. Indeed, since 2015 Spain has experienced an extraordinary chain of interim governments with General Elections in April and again in November 2019. In January 2020, a coalition government has been conformed and it has to seek deals to adopt the measures and implement the policies needed to comply with SDG. As it has been exposed, the 2030 Agenda has a leading presence in a wide range of institutional actions plans and formal declarations, but action to positive measures is also required. On top of everything, the changes needed are neither minor nor easy to put in force.

The new government has already shown concern, and a Declaration facing Climate and Environmental Emergency has been approved by the Cabinet, to implement 30 lines of actions. Among the first to be launched, within the first 100 days in office, there is a draft bill to achieve zero net emissions by 2050, establish the basis for decarburization, develop the second National Climate Change Adaptation Plan and drive the transformation of the industrial and service model through the formalization of Fair Transition Agreements. Other measures to be developed in a near future engage the integration of the effects of climate change on economic, consumer, mobility, housing, international cooperation, education or health policies.

In short, a turnaround is essential for Spain to achieve the objectives set out in the 2030 Agenda. The first steps taken by the new government, on the long and difficult road to fulfill the objectives, seem hopeful and prove a mounting concern about the situation.

We hope that the results obtained and the conclusions presented in this paper would be useful for the governmental authorities to become aware of the situation and adopt legislation and policies that will allow a reorientation. The aim is also to raise awareness among citizens of the urgent need for a paradigm shift.

Author Contributions: Conceptualization, data curation, formal analysis, methodology, review and editing of manuscript, A.B.-Á. and R.G.-F. All authors have read and agreed to the published version of the manuscript.

Funding: This research received no external funding.

Acknowledgments: The authors truly thank University of Oviedo support and all the suggestions and concerns expressed by the peer reviewers and the editor, which have improved the final result.

Conflicts of Interest: The authors declare no conflicts of interest.

\section{References}

1. Raszkowski, A.; Bartniczak, B. On the Road to Sustainability: Implementation of the 2030 Agenda Sustainable Development Goals (SDG) in Poland. Sustainability 2019, 11, 366. [CrossRef]

2. Firoiu, D.; Ionescu, G.H.; Băndoi, A.; Florea, N.M.; Jianu, E. Achieving Sustainable Development Goals (SDG): Implementation of the 2030 Agenda in Romania. Sustainability 2019, 11, 2156. [CrossRef]

3. Durán y Lalaguna, P. The sustainable development goals. In International Society and Sustainable Development Goals; Editorial Aranzadi: Pamplona, España, 2016; pp. 35-48, ISBN 978-84-9135-466-6.

4. United Nations Resolution 70/1. Transforming Our World: The 2030 Agenda for Sustainable Development, Adopted by the General Assembly on 25 September 2015. Available online: https://undocs.org/en/A/RES/70/1 (accessed on 20 August 2019).

5. European Commission the 2030 Agenda for Sustainable Development and the SDGs. Available online: https: //ec.europa.eu/environment/sustainable-development/SDGs/index_en.htm (accessed on 12 December 2019).

6. Dörgő, G.; Sebestyén, V.; Abonyi, J. Evaluating the Interconnectedness of the Sustainable Development Goals Based on the Causality Analysis of Sustainability Indicators. Sustainability 2018, 10, 3766. [CrossRef] 
7. Diaz-Sarachaga, J.M.; Jato-Espino, D.; Castro-Fresno, D. Is the Sustainable Development Goals (SDG) index an adequate framework to measure the progress of the 2030 Agenda? Sustain. Dev. 2018, 26, 663-671. [CrossRef]

8. Fourie, W. Aligning South Africa's National Development Plan with the 2030 Agenda's Sustainable Development Goals: Guidelines from the Policy Coherence for Development movement. Sustain. Dev. 2018, 26, 765-771. [CrossRef]

9. Consejo de Ministros de España Acuerdo de aprobación del "Plan de Acción para la Implementación de la Agenda 2030: Hacia una Estrategia Española de Desarrollo Sostenible". Available online: https://www.lamoncloa.gob.es/consejodeministros/Paginas/enlaces/290618-agenda2030.aspx (accessed on 12 December 2019).

10. Ministerio de la Presidencia, Relaciones con las Cortes e Igualdad Orden PCI/169/2019, de 22 de febrero, por la que se crea el Consejo de Desarrollo Sostenible. Available online: https://www.agenda2030.gob.es/sites/default/files/recursos/CONSEJO\%20DE\%20DESARROLLO\% 20SOSTENIBLE\%20\%28BOE\%2022.02.19\%29.pdf (accessed on 9 January 2019).

11. Gobierno de España Spain's Report for the 2018 Voluntary National Review. Available online: https://sustainabledevelopment.un.org/content/documents/203295182018_VNR_Report_Spain_EN_ ddghpbrgsp.pdf (accessed on 12 December 2019).

12. Sánchez Pérez-Castejón, P. Official Transcription of the Appearance. Available online: https://www.lamoncloa. gob.es/presidente/intervenciones/Paginas/2019/prsp25092019bis.aspx (accessed on 12 December 2019).

13. Gobierno de España; Ministerio de Sanidad, Consumo y Bienestar Social Estrategia nacional de prevención y lucha contra la pobreza y la exclusión social 2019-2023. Available online: https://www.mscbs.gob.es/ssi/familiasInfancia/inclusionSocial/inclusionSocialEspana/Estrategia_ Prev_y_Lucha_Pobreza_2019-23.pdf (accessed on 12 December 2019).

14. Gobierno de España; Ministerio para la Transición Ecológica Marco Estratégico de Energía y Clima: Una oportunidad para la modernización de la economía española y la creación de empleo. Available online: https://www.miteco.gob.es/es/cambio-climatico/participacion-publica/marco-estrategico-energiay-clima.aspx (accessed on 12 December 2019).

15. Gobierno de España; Ministerio de Fomento Agenda Urbana Española. Available online: http://www.aue. gob.es/ (accessed on 12 December 2019).

16. Gobierno de España Campaña \#ODSéate. Available online: https://www.agenda2030.gob.es/es/campanaodseate (accessed on 12 December 2019).

17. Prieur, M. Les indicateurs juridiques. Outils d'évaluation de l'effectivité du droit de l'environnement; Institut de la Francophonie pour le Développement Durable (IFDD): Québec, QC, Canada, 2018; ISBN 978-2-89481-266-2.

18. European Commission Communication COM (2016) 739 Final from the Commission to the European Parliament, the Council, the European Economic and Social Committee and the Committee of the Regions "Next Steps for a Sustainable European Future". Available online: https://eur-lex.europa.eu/legal-content/ EN/TXT/?uri=COM\%3A2016\%3A739\%3AFIN (accessed on 21 August 2019).

19. Terama, E.; Milligan, B.; Jiménez-Aybar, R.; Mace, G.M.; Ekins, P. Accounting for the environment as an economic asset: Global progress and realizing the 2030 Agenda for Sustainable Development. Sustain. Sci. 2016, 11, 945-950. [CrossRef] [PubMed]

20. Jomo, K.S.; Chowdhury, A.; Sharma, K.; Platz, D. Public-Private Partnerships and the 2030 Agenda for Sustainable Development: Fit for Purpose? Available online: https://www.un.org/esa/desa/papers/2016/ wp148_2016.pdf (accessed on 9 January 2020).

21. Bodenstein, T.; Faust, J.; Furness, M. European Union Development Policy: Collective Action in Times of Global Transformation and Domestic Crisis. Dev. Policy Rev. 2017, 35, 441-453. [CrossRef]

22. Hackenesch, C.; Kloke-Lesch, A.; Koch, S.; Niestroy, I.; Scholz, I. Towards a "Sustainable Development Union": Why the EU Must Do More to Implement the 2030 Agenda. Available online: https://www.die-gdi.de/en/briefing-paper/article/towards-a-sustainable-development-union-whythe-eu-must-do-more-to-implement-the-2030-agenda/ (accessed on 9 January 2020).

23. European Union Agency for Fundamental Rights Implementing the Sustainable Development Goals in the EU: A Matter of Human and Fundamental rights. Available online: https://fra.europa.eu/sites/default/files/ fra_uploads/fra-2019-fundamental-rights-report-2019-focus_en.pdf (accessed on 9 January 2020). 
24. European Parliament Resolution of 12 May 2016 on the Follow-Up to and Review of the 2030 Agenda (2016/2696(RSP)). Available online: https://eur-lex.europa.eu/legal-content/EN/TXT/?uri=CELEX\% 3A52016IP0224 (accessed on 9 January 2020).

25. European Parliament Resolution of 6 July 2017 on EU Action for Sustainability (2017/2009(INI)). Available online: https://eur-lex.europa.eu/legal-content/EN/TXT/?uri=CELEX\%3A52017IP0315 (accessed on 9 January 2020).

26. Council of the European Union Council Conclusions on A Sustainable European Future: The EU Response to the 2030 Agenda for Sustainable Development, 10370/17. Available online: https://www. unodc.org/documents/brussels/ABOUT\%20EU\%20/2017.06_EU_RESPONSE_2030AGENDA.pdf (accessed on 9 January 2020).

27. Council of the European Union Council Conclusions "Towards an ever more sustainable Union by 2030", 8286/19. Available online: https://data.consilium.europa.eu/doc/document/ST-8071-2019-INIT/en/pdf (accessed on 9 January 2020).

28. European Commission Reflection Paper: Towards a Sustainable Europe by 2030. Available online: https: //ec.europa.eu/commission/sites/beta-political/files/rp_sustainable_europe_30-01_en_web.pdf (accessed on 9 January 2020).

29. Derr, K.; Dirth, E.; Hege, E.; Niestroy, I.; Zondervan, R.; European Parliament; Directorate-General for External Policies of the Union. Europe's Approach to Implementing the Sustainable Development Goals: Good Practices and the Way Forward; European Parliament: Brussels, Belgium, 2019; ISBN 978-92-846-4578-7.

30. Kanuri, C.; Aromar, R.; Espey, J.; Kuhle, H. Getting Started with the SDGs in Cities: A Guide for Stakeholders. Available online: https://irp-cdn.multiscreensite.com/be6d1d56/files/uploaded/9.1.8.-Cities-SDG-Guide.pdf (accessed on 9 January 2020).

31. European Commission. Subgroup on "Delivering SDGs at Local and Regional Level" Recommendations to the European Commission by the Subgroup on "SDGs at Local and Regional Level" of the Multi-Stakeholder Platform on the Implementation of the Sustainable Development Goals in the EU. Available online: https: //ec.europa.eu/info/sites/info/files/delivering-sdgs-local-regional-level.pdf (accessed on 9 January 2020).

32. Levarlet, F.; Celotti, P.; Alessandrini, M. A Territorial Approach for the Implementation of the SDGs in the EU-The role of the European Committee of the Regions. Available online: https:/op.europa. eu/en/publication-detail/-/publication/21866ea2-574c-11e9-a8ed-01aa75ed71a1/language-en (accessed on 9 January 2020).

33. European Commission EU SDG Indicator set 2019. Result of the Review in Preparation of the 2019 Edition of the EU SDG Monitoring Report. Available online: https://ec.europa.eu/eurostat/documents/276524/ 9479054/2019-01-08_EU_SDG_indicator_set_2019_review_final_report.pdf/7234d06f-4fd5-40ce-80717bcddc4013c2 (accessed on 21 August 2019).

34. Webster, N.; Ravnborg, H.M. Monitoring the implementation of the Sustainable Development Goals. The Role of the Data Revolution. Available online: https://op.europa.eu/en/publication-detail/-/publication/9d7c4d269aeb-11e6-868c-01aa75ed71a1/language-en (accessed on 9 January 2020).

35. Davis, K.; Fisher, A.; Kingsbury, B.; Engle Merry, S. Governance by Indicators: Global Power through Classification and Rankings; Oxford University Press: Oxford, UK, 2012; ISBN 978-0-19-965824-4.

36. Miola, A.; Schiltz, F. Measuring sustainable development goals performance: How to monitor policy action in the 2030 Agenda implementation? Ecol. Econ. 2019, 164, 106373. [CrossRef] [PubMed]

37. Ordaz, E. The SDGs Indicators: A Challenging Task for the International Statistical Community. Glob. Policy 2019, 10, 141-143. [CrossRef]

38. Guijarro, F.; Poyatos, J. Designing a Sustainable Development Goal Index through a Goal Programming Model: The Case of EU-28 Countries. Sustainability 2018, 10, 3167. [CrossRef]

39. Meurs, M.; Seidelmann, L.; Koutsoumpa, M. How healthy is a 'healthy economy'? Incompatibility between current pathways towards SDG3 and SDG8. Glob. Health 2019, 15, 83. [CrossRef] [PubMed]

40. Pradhan, P.; Costa, L.; Rybski, D.; Lucht, W.; Kropp, J.P. A Systematic Study of Sustainable Development Goal (SDG) Interactions. Earths Future 2017, 5, 1169-1179. [CrossRef]

41. Weitz, N.; Carlsen, H.; Nilsson, M.; Skånberg, K. Towards systemic and contextual priority setting for implementing the 2030 Agenda. Sustain. Sci. 2018, 13, 531-548. [CrossRef] [PubMed] 
42. Wieck, C.; Hausmann, I. Indicators Everywhere: The New Accountability of Agricultural Policy? Available online: https://ageconsearch.umn.edu/record/289722/files/Wieck-Indicators\%20everywhere-136_a. pdf (accessed on 9 January 2020).

43. Ali, M. The China-Pakistan Economic Corridor: Tapping Potential to Achieve the 2030 Agenda in Pakistan. China Q. Int. Strateg. Stud. 2018, 04, 301-325. [CrossRef]

44. Pineda-Escobar, M.A. Moving the 2030 agenda forward: SDG implementation in Colombia. Corp. Gov. Int. J. Bus. Soc. 2019, 19, 176-188. [CrossRef]

45. López Pagán, J. La Agenda 2030 en Iberoamérica: Visión y misión desde el ámbito local. Comillas J. Int. Relat. 2019, 16, 138-153. [CrossRef]

46. MacFeely, S. The Big (data) Bang: Opportunities and Challenges for Compiling SDG Indicators. Glob. Policy 2019, 10, 121-133. [CrossRef]

47. Bouckaert, G.; Loretan, R.; Troupin, S. Public Administration and the Sustainable Development Goals. 2016-03 Session of the United Nations Committee of Experts on Public Administration. Available online: https://lirias.kuleuven.be/retrieve/389289 (accessed on 9 January 2020).

48. Instituto Nacional de Estadística Nota metodológica Indicadores de la Agenda 2030 para el Desarrollo Sostenible. Available online: https://www.ine.es/ods/metodologia_ods.pdf (accessed on 22 August 2019).

49. Eurostat Sustainable Development Goals—Overview. Available online: https://ec.europa.eu/eurostat/web/ sdi/overview (accessed on 20 September 2019).

50. Eurostat Sustainable Development Goals-Main Tables. Available online: https://ec.europa.eu/eurostat/web/ sdi/main-tables (accessed on 20 September 2019).

51. Jibrilla, A. Forecasting FDI Inflows from the United States (USA) to Nigeria over Ten Years Period. Int. J. Econ. Financ. Manag. 2018, 3, 2545-5966.

52. Canela, M.Á.; Alegre, I.; Ibarra, A. Holt-Winters Forecasting. In Quantitative Methods for Management; Springer International Publishing: Cham, Switzerland, 2019; pp. 121-128, ISBN 978-3-030-17553-5.

53. Held, B.; Moriarty, B.; Richardson, T. Microsoft Excel Functions and Formulas, 5th ed.; Mercury Learning and Information: Dulles, VA, USA, 2019; ISBN 978-1-68392-373-2.

54. Baltac, A.-G. Economic and Financial Analysis Based on Time Series Method. Int. J. Acad. Res. Account. Financ. Manag. Sci. 2015, 5, 77-82. [CrossRef]

55. Box, G.E.P.; Jenkins, G.M.; Reinsel, G.C.; Ljung, G.M. Time Series Analysis: Forecasting and Control, 5th ed.; Wiley Series in Probability and Statistics; John Wiley \& Sons, Inc.: Hoboken, NJ, USA, 2016; ISBN 978-1-118-67502-1.

56. Kangas, O.; Simanainen, M.; Honkanen, P. Basic Income in the Finnish Context. Intereconomics 2017, 52, 87-91. [CrossRef]

57. Boto Álvarez, A. El reto del bienestar en tiempos de ruina: Medidas de fomento de la vivienda social en Francia ante la crisis inmobiliaria. Rev. Gen. Derecho Adm. 2013, 33, 1-9.

58. Fernández-Rodríguez, E.; García-Fernández, R.; Martínez-Arias, A. Influence of Ownership Structure on the Determinants of Effective Tax Rates of Spanish Companies. Sustainability 2019, 11, 1441. [CrossRef]

59. Fernández-Rodríguez, E.; García-Fernández, R.; Martínez-Arias, A. Business and institutional determinants of Effective Tax Rate in emerging economies. Econ. Model. 2020. [CrossRef]

60. European Training Foundation Making Labour Markets More Inclusive for Workers and Potentially Vulnerable Groups. Available online: https:/ec.europa.eu/social/BlobServlet?docId=20974\&langId=en (accessed on 28 December 2019).

61. Gobierno de España; Ministerio de Agricultura, Pesca y Alimentación La agricultura ecológica en España. Available online: https://www.mapa.gob.es/en/alimentacion/temas/produccion-ecologica/default_antigua. aspx (accessed on 28 December 2019).

62. Rincón, V.; González, M.; Barrero, K. Women and leadership: Gender barriers to senior management positions. Intang. Cap. 2017, 13, 319. [CrossRef]

63. Gobierno de España; Ministerio de Industria, Turismo y Comercio Plan de Acción Nacional de Energías Renovables de España (PANER) 2011-2020. Available online: https://energia.gob.es/desarrollo/ EnergiaRenovable/Documents/20100630_PANER_Espanaversion_final.pdf (accessed on 28 December 2019).

64. OECD. OECD Employment Outlook 2019: The Future of Work; OECD Employment Outlook; OECD: Paris, France, 2019; ISBN 978-92-64-72715-1. 
65. Gobierno de España; Ministerio de Industria, Energía y Turismo; Ministerio de Hacienda y Administraciones Públicas Administraciones Públicas Agenda Digital para España. Available online: https://avancedigital.gob.es/planes-TIC/agenda-digital/DescargasAgendaDigital/Plan-ADpE_ Agenda_Digital_para_Espana.pdf (accessed on 28 December 2019).

66. Blanco, A. Informe España 2018; Universidad Pontificia Comillas, Cátedra José María Martín Patino de la Cultura del Encuentro: Madrid, Spain, 2018; ISBN 978-84-8468-769-6.

67. United Nations Economic and Social Council Special Edition: Progress towards the Sustainable Development Goals. Report of the Secretary-General (E/2019/68). Available online: https://unstats.un.org/sdgs/files/report/ 2019/secretary-general-sdg-report-2019--EN.pdf (accessed on 6 March 2019).

C 2020 by the authors. Licensee MDPI, Basel, Switzerland. This article is an open access article distributed under the terms and conditions of the Creative Commons Attribution (CC BY) license (http://creativecommons.org/licenses/by/4.0/). 